\title{
II. Nürnbergische Geheimschrift in 15. und zu Anfang des 16. Jahrhunderts.
}

\author{
Von \\ Dr. F. W a g ner, \\ Gymnasiallehrer in Berlin.
}

I.

Gegenüber den Besitzungen der fränkischen Hohenzollern befand sich die freie Reichsstadt Nüruberg bei Ausgang des 15. Jahrhunderts in einer eigenartigen geographischen Lage. 1) Sie war ringsum fast rollständig eingeschlossen von dem fürstlichen Gebiete, und die meisten ihrer Verbindungsstrassen mit der Aussenwelt waren für den Fall, dass Feindseligkeiten ausbrachen, abgeschnitten. Beinahe günstiger noch war es für die Bürger, wenn offen und ehrlich Krieg geführt wurde, als weun ein Zustand ungewissen Friedens und heimlicher Begünstigung erklärter Feinde Nürnbergs eintrat. Denn im ersteren Falle reichten die Machtmittel der Stadt aus, um gewaltsam den nicht ganz geschlossénen Ring zu durchbrechen und Fühlung mit näheren oder entfernteren Freunden zu gewinnen; andernfalls aber konnte man sich nur schwer gegen Schaden sichern, und nicht blos der Handel litt dabei gewaltig, sondern auch der Verkehr mit den andern Reichsstädten, mit den benachbarten freundlich gesinnten Fürsten, mit dem Reiche war gefährdet und zeitweilig unterbrochen. Und nachdem Albrecht Achill in dem grossen Städtekriege vergeblich versucht hatte, mit seiner Heldenkraft die freiheitsliebende Bürgergemeinde niederzuringen, begann

1) Sie war, wie ein Bericht Nünberger Gesandten vom 23. April 1500 es ausdrackt, mit den Hohenzollern "gemengt". Vgl. dazu die Karte im 11. Bande der deutschen Stadtechroniken. 
in eigenthümlicher, viele Jahrzehnte hindurch dauernder, stillerh emeist diplomatisch geführter Kampf zwischen den Hofe zu Ansbac. und der Reichsstadt. Bald nach dem Tode des brandenburgischen Kurfürsten (1486) nahmen die $\mathrm{ihm}$ in den fränkischen Ländern nachfolgenden Söhne, die Markgrafen Friedrich und Sigismund, die früheren Streitigkeiten wieder auf. Zuerst suahten sie sich den Rücken $z u$ decken. Sie gewannen (1488) - durch reichliche Unterstützung in den Niederlanden - die Gunst Kaiser Friedrichs III. und. seines Sohnes Maximilian und damit den Befehl zur Wiederaufrichtung des Landgerichtes. Ganz besonders vortheilhaft für sie war aber die Gründung des schwäbischen Bundes und ibre Aufnahme in denselben. ( $\nabla$ gl. darüber meiue Abhandlung: Die Aufnahme der fränkischen Hohenzollern in den schwäb. Bund. Progr. d. K. Friedr. Wilh Gymn. zu Berlin 1880 und die Fortsetzung in d. Forsch. z. deutsch. Gesch. XXII. S. 261-327.) Anfänglich nahmen die Misshelligkeiten zwischen den Bundesmitgliedern und den Herzögen Albrecht IV. von Bayern-München und Herzog Georg dem Reichen von BayernLandshut die ganze Aufmerksamkeit und Thätigkeit, wie der andern Nachbarfürsten, so auch der beiden Hohenzollern in Anspruch. Erst nach der Demüthigung der bayerischen Partei, auf deren Seite übrigens auch Nürnberg gestanden hatte, so dass es mittelbar wenigstens von dieser Niederlage mitbetroffen wurde (1492), tritt seitens der Markgrafen deutlich das Bestreben hervor, den schwäbischen Bund gegen Nürnberg zu verwerthen. Gründe zu Klagen gab es auf beiden Seiten beständig, wenn sie auch häufig nur ganz geringfügige Sachen betrafen. Bald wurden Beschwerden Brandenburgs über Nürnberg ein stetig wiederkehrender Punkt der Bundesverhandlungen. Daneben wurden dann noch anderweitige Versuche, eine gütliche Einigung herbeizuführen, freilich meist erfolglos, gemacht. Unermüdlich war anfangs der Erzbischof Berthold von Mainz, die Hadernden zu versöhnen. Dann nahm sich Herzog Albrecht der Beherzte von Sachsen der Streitigkeiten an, und wirklich glückte es, nach einem im J. 1493 unternommenen vergeblichen Versuche und nachdem es anfangs 1494 beinahe zu offenen Feindseligkeiten gekommen war, 1496 dem sächsischęn Rathe Dietrich von Harras, mehr einen Waffenstillstand als einen Frieden herbeizuführen. Bald begannen die Reibungen von neuem. Schon am 1. Oktober 1498 finden sich wieder Nürnbergische Gesandte am Hoflager des Markgrafen in Ansbach ein, um neuerdings ausgebrochene Misshellig- 
keiten beizulegen. Beiderseits bemüht man sich offenbar, einen recht gemässigten Ton zu wabren; die Gesandten der Stadt werden zur markgräflichen Tafel gezogen. Aber sachlich erwies sich eine Uebereinkunft doch nicht möglich. Unverrichteter Dinge kehren dic Gesandten heim, und von Tage zu Tage wird nun das Verhältniss wieder gereizter. Im März 1499 erbietet sich der Rath zu Nürnberg noch einmal, ein Schiedsgericht annehmen zu wollen; aber bereits war die Kugè im Rollen und nicht mehr aufzuhalten. Markgraf Friedrich suchte den Krieg gegen die Schweizer in äbnlicher Weise für sich auszubeuten, wie vor 11 Jahren den niederländischen Feldzug. Er wusste, dass er dem König Maximilian, wie dem scbwäbischen Bunde im Felde unentbehrlich sei. Nichtsdestoweniger gab er sich den Anschein, in die Heimath aufbrechen zu müssen, weil er von neuen Feindseligkeiten der Nürnberger, Erbauurg von Thürmen ausserhalb der Stadtmauer; gehört habe. Vorläufig erreichte er seinen Zweck. Um ihn zum Bleiben zu bewegen, wurde ihm Bundeshilfe gegen Nürnberg in Aussicht gestellt. Als er freilich nach Beendigung des Schweizerkrieges den Bund an das gegebene Versprechen mahnte, fanden sich Ausflüchte. Maximilian, der Nürnbergs Geldhilfe ebenso nöthig brauchte wie die Truppen des Markgrafen, zog die Sache an sich, und auf dem Reichstage zu Augsburg (1500) begannen weitläufige Verhandlungen. Im Nürnberger Archiv befinden sich nun die Berichte der städtischen Gesandten darüber; sie liefern ein anschauliches und lehrreiches Bild von den damaligen Zuständen. Aber sie bieten dem Leser dadurch viele Schwierigkeiten, dass dunkle. Stellen darin vorkommen, die sich wunderlich genug ausnehmen. So heisst es z. B. in der Relation rom 25. April 1500: als ich, Anthoni Tetzel, e. w. bevelh nach die amsel heut fru hab vernagelt, hat sie solchs gar zu grossem dank angenomen mit fast hohem erpieten solchs zu verdienen; die amsel hat auch mir, Anthoni Tetzel, daneben und alspald angezaigt, das sich pfab gestern freitag zum frumal zu ir, der amsel, hette geladen u.s. w. In demselben Schriftstück ist von einem zeislin, sperber, auerhahn die Rede. Oder in dem Bericht vom 2. Mai 1500 findet sich die Stelle: in dieser handlung hat die a msel so suss gesungen; ob es ain nachtigal het getan, so wer es doch genug und ist möglich, sie hab ir kelen mit den amaisaiern also erfrist; wiewol wir dannocht on das kain mistrost in sie setzen, dann aus rechter musica ze singen. So schwingt das zeislin sein gefider 
auch in aller höhe, hat auch bei uns sein schnebelein aus berelh des sperbers darinnen wol und getreulich gewetzt. - In einem Schreiben vom 22. Mai wird gesagt: item ron wegen des paums haben wir sovil fleis gepraucht, das uns desselben sind abschrift zu nemen gestattet, desgleichen der handlung und recess zu Esslingen der merertail etc. In ähnlicher Weise laufen durch die ganze Korrespondenz Bezeichnungen, die nicht im eigentlichen Wortsinne rerstanden werden sollten. Neben Sperber, Amsel, Zeisig, A uerhahn und Pfau (=Pfab) treten Falke, Blaufuss, Waldvogel, Haselhuhn, Kohlmeise, Eichborn, Sittich, Salamander und Stieglitz auf; mehrfach ist von dem Mannne oder den Verwandten mit goldnen Sporen die Rede. Der Baum und seine Aeste werden oft erwähnt; einmal ist auch die Bezeichnung die rom schwarzen erdreich gebraucht. Die Ausdrücke nägel, vernageln müssen dem Zusammenhange nach auch eine uneigentliche Bedeutung haben. Es ist klar, dass die Gesandten, nicht um ihren Witz zu beweisen, sondern infolge einer bestimmten Verabredung mit ihren Auftraggebern sich dieser Geheimsprache bedient haben, und auch der Grund für diese Einrichtung tritt deutlich genug in dem Briefwechsel hervor: man fürchtet beiderseits, im Rathe zu Nürnberg sowobl wie im Schoosse der Nürnbergischen Gesandtschaft zu Augsburg, dass die überaus wiehtigen und viele diplomatische Geheimnisse enthaltenden Briefschaften vom Feinde zufgefangen und zu seinen $Z$ wecken verwerthet werden könnten. 1) Es sollte nun durch Anwendung dieser Geheimsprache das Verständniss erschwert und für alle Fälle diesen Mittheilungen die unwiderlegliche Beweiskraft bei etwaigen Anklagen genommen werden.

Es frägt sich nun, ob diese geheimen Zeichen zu enträthseln sind oder nicht. Bei einigen ist es für den in die Geschichte dieser Zeit einigermassen Eingeweihten leicht hinter das Geheimniss

1) In dem Bericht vom 4. Juni 1500 heisst es in dieser Beziehung: wir haben e. w. hievor geschriben, das ir mögt ausnemen, wen ir wollt; doch mit unterschid, das der federn nit zu bevelhen sei. Der federu halben ist darumb geschehen, ob der briefe wer nidergelegen ..... Gleich nach ihrer Ankunft in Augsburg schrieben die Gesandten am 4. April: als vor unserm ahschid von ainer postrey etwas reden sind bescheen, bedunkt uns gut und notturftig sein, dieselben z.wuschen hie und Nurmberg an gelegen end uffzerichten .... Und am 19. März 1500 heisst es: wir haben e. w. die schriften zwifach zugeschickt; ob aine underwegen niderleg, hoffen wir, die ander e. w. zukomen werde. 
zu kommen. Dass mit dem Sperber König Maximilian, mit den Pfau (pfab) der Markgraf Friedrich von Brandenburg, mit dem Baume und seinen Aesten der schwäbische Bund und seine Mitglieder gemeint seien, ergiebt sich aus dem Zusammenhange ohne grosse Schwierigkeit. Bei andern Bezeichnungen aber zeigt erst die Vergleichung verschiedener Stellen, welche Person unter dem Thiernamen zu verstehen ist. Man strüubt sich fast, unter der Amsel den Erzbischof Berthold ron Mainz zu errathen; denn zu dem Bilde, das Ranke ron ihm entworfen hat, wollen die Mittheilungen in diesen Berichten gar nicht recht passen. Die A m sel ist "vern a ge lt" worden, heisst nänlich nichts anderes, als Berthold hat Geldgeschenke von den Nürnberger Gesandten erhalten, und zwar ist er nicht einmal, sondern mindestens zweimal bestochen worden. 1) Und doch kann kein $Z$ weifel darüber obwalten, dass mit der Amsel der Erzbischof gemeint ist. Es ergiebt sich dies nicht nur aus dem Znsammenhange, sondern auch aus einer Randbemerkung, die sich in einem der Berichte findet. Diese Relationen wurden jedenfalls im Rathe vorgelesen. Zu diesem Behufe wurden sie aber erst zubereitet. Es gab Stellen, die sich gar nicht zur Mittheilung eigneten. Bei diesen steht am Rande tace. Wahrscheinlich sollte nun aber die Bedeutung der Geheimzeichen auch nicht allgemein bekannt werden und deshalb mochten der Rathsversammlung nur die richtigen Namen vorgelesen werden. ${ }^{2}$ ) Um dem Vorleser dies zu erleichtern, findet sich in ejnigen Berichten für diese oder jene Bezeichnung in margine die Bedeutung vermerkt. ${ }^{3}$ ) Daraus ergiebt

1) Um die Bedeutung der nägel und des vernageln sicher zu stellen, führe ich ausser der obigen noch einige Stellen an. Bericht vom 22. Juni 1500: so baben wir auch noch anf ain weg gedacht, ob bei dem Sperber were anzuhalten, das er die sach bei dem ersten gegeben recess bleiben und der streng und ernstlich mandat ausgeen liess an dem Pfaben, demselben zu gleben und mit der gewaltsam still zu steen und das e. w. desselben durch verschreibung versichert, damit es des andern tags nit widerumb geendert wurde und das man dem Sperber darum ein ermel mit 3 oder $400 \mathrm{n}$ e g e l i n anheftet... Bericht rom 13. Mai : salamander habe ich noch nicht vernagelt, weil sein junger nit hie ist, der bei der sache gut were und aus furcht, es sei nach gelegenheit seins'vorforderen zu wenig.... Bericht vom 12 . April : so sein wir n e gel notturftig; der wolle uns e. w. 2000 furderlich her gen Augspurg bestellen; dann wir hie nit $\mathrm{n}$ e gel durch wechsel mogen aufpringen ....

8) Christoph Scheurl schreibt 1516: Reliqui (Mitglieder des kleinen Rathes) in comparatione horum (der 7 Elteren Herren) parum sciunt etc.

s) Von besonderer Wichtigkeit für die oben dargestellten Verhältnisse 
sich mit Sicherheit, dass unter der Amsel der Erzbischof von Mainz zu verstehen ist. Leider lassen sich nicht alle Zweifel so leicht lüsen wie dieser. Besonders für die weniger oft auftretenden oder minder bedeutenden Persünlichkeiten fehlt dieser Schlüssel 1), und gerade bei ihnen giebt auch der \%usammenhang nicht inmer genügenden Anhalt, ihre Identität festzusteHen. Auf Seiten der Nürnberger stehlt der Falke. Dass damit Herzog Albrecht IV. von München gemeint sei, zeigt die Stelle im Bericht vom 26. Mai: Wolf von Ahaim, des falk e n hofmeister (vgl. Müller, Reichstagsth. unter Maximilian II. 684); Marginalbemerkungen bestätigen es. Ebenso gehört zu den Freunden der Nürnberger der bei Beginn des Reichtags öfters erwähnte Sittich. In dem Bericht vom 22. Mai wird ein Verhör der Parteien vor dem Könige erwähnt; da sitzen zur Rechten: am sel, eich horn, salamander, has elhuhn; zur Linken: waldvogel, falk, sittich und Meklenburg. Daraus geht wenigstens soviel herror, dass der Sittich unter den weltlichen Fürsten sitzt, und der Umstand, dass er neben dem Falken seinen Platz hat, deutet auf einen Wittelsbacher hin. In dem Bericht von 8. Mai wird erwähnt, dass der Rentmeister zur Weiden „von wegen Clement von Wisentau" an den Rath von Nürnberg geschrieben habe ${ }^{2}$ ) und deshalb den Gesandten befohlen worden sei, mit den Sittich zu verhandeln. Aus dieser Notiz folgt doch wohl, dass Herzog Georg von Bayern-Landshut unter dem Sittich

sind die Berichte vom 2. und 5. Mai 1500. In diesen wechseln die Anmerkungen tace und lege mit einander $a b$. So $z$. B. steht tace neben der oben angefuhrten Stelle: in dieser handlung etc. Bei Amsel ist in dem Bericht vom 5. Mai immer Erzbischof von Mainz darüber geschrieben. Im Bericht vom 4. Juni ist für amsel am Rande bemerkt: ein gewaltiger.

1) Dass ein wirklicher Schlüssel sich sowohl in Nürnberg wie im Besitz der Gesandten befand, ergiebt sich wohl mit Sicherheit aus den weiter unten folgenden Mittheilungen sowie auch aus der im Bericht vom 15. April ausgesprochenen Bitte : Item nachdem unser gn. h. von Mentz so getreulich in uusern sachen handelt, wolle uns $e$. $w$. versteen lassen, ob und wie $s . f$. g. zu vereren sei, wollet uns auch eine copei von dem v oc a b u l a ri furderlich schicken. Dadurch wird auch erklärlich, dass erst einige Zeit nach diesem Ersuchen (also nach dem Eintreffen des Vokabulariums) die Geheimzeichen zahlreicher und fast regelmäsaig in den Berichten auftreten.

) In einem andern Aktenfaszikel findet sich (vom 20. April 1500) dieser Brief des Rentmeisters zur Weiden Hans von Floss an den Rath zu Nürnberg, dass dessen Diener Clemens von Wiesenthau ihm anstatt seines Herrn, des Herzogs Georg von Bayern, noch keine Rechnung gelegt habe. 
zu verstehen ist. Dafür spricht auch die folgende Stelle im Bericht rom 13. Mai: under denen haben wir dem sittich die sach gar wol geteutscht und angezaigt, wa es zum ernst oder tat langen, das sein darinnen nicht verschont, sunder er auch in die hilf gezogen wurde. Der hat uns auch geraten, bei der ko. mt. statlich anzuhalten und in dem beschid nit zu grubeln, und sich furwar so gnedig bewisen, das wir ain trost und freud darab haben empfangen und sich erpoten, dise ding mit ko. mt. selbs zu handeln ..... e. w. vorfarn und ir hettens auch unb seinen vater ${ }^{1}$ ) und ine wol verdint. . . . Denn offenbar bezieht sich diese Ankündigung auf das Bündniss, in dem Herzog Georg mit Nürnberg stand. 2) Noch sicherer freilich würde die Identität festzustellen sein, wenn sich nachweisen liesse, dass er mit Nürnberg wegen Jueynburg im Streit gelegen habe. Doch vermag ich darüber nichts beizubringen.

Als sehr einflussreich wird unter den Gönnern Nürnbergs der Waldrogel (oder auch die Waldvögel) erwähnt. Er sitzt liber den bayerischen Fürsten, wenn Verhör gehalten wird. Schon daraus könute man wohl darauf schliessen, dass es ein Kurfüst sein möchte. Am 15. Juni berichten die Gesandten: zuerst sei der $P$ fab (d. h. der Markgraf von Brandenburg) gehört worden; dann seien sie vorgefordert worden und hätten gebeten, ihnen das Vorbringen des Gegners zu eröffnen. Darauf habe sich der König mit der Amsel und dem Waldvogel, $z$ wischen denen ergesessen habe, besprochen. Noch wichtiger für die Feststellung dieser Persönlichkeit ist folgende Stelle im Bericht vom 4. Juli: So hat waldvogel heut dis tags

1) Jedenfalls geht diese Aeusserung auf das Bündniss Nürnbergs mit (jeorgs Vater Ludwig im Kriege gegen Albrecht Achill im J. 1461.

2) Dieses Bundniss machte den Nürnberger Gesandten Bedenken, als es sich um den Fintritt ibrer Vaterstadt in den schwabischen Bund handelte. Sie schreiben am 4 Juni : Nun ist des pa u ms ordnung auf den ko. landfriden und zu handhabung desselben ergrundet und gestellt. Das haben alle fursten, dem p a n m verwaindt, die on zweifel mit andern auch in pundnus sind, bedacht und ganz kein ausnemen gethan. Der $f \mathrm{alk}$ hat auch eu nit ausgenommen. Darumb haben wir in vorigem schreiben gesetzt die meinung; wa aber e. w. niemand wolt augnemen, das mocht auch geschehen und dannoch unverletzt aller pillichait etc. Dann e. w. nemen aus oder nit, so gilt es des b a u m s halben obverlauter ursachen halben gleich. Wolt aber e. w. je gelieben eur pundtgenossen auszunemen, so bedunkt uns zu thun sein, das ir die Bayrischen het ausgenomen die Zeit aus, dieweil eur ainung weret. ..... 
auch nach mir geschickt und vertreulich mit mir allein in einom gemach hei 2 stunden lang gehandelt und ich in die handlung entdeckt, des er dannocht des pfaben halben verwundern und vorhin nit wissen gehabt. Bei dem find ich gnad und gunst und das er e. w. sach nit verunglimpft, dann allein die that $\mathrm{zu}$ Ried. die will er nit loben und zeigt sich sorgfeltig an als ein hoch verstendiger furst; wa die sach lang in dem wesen bleib steen, das es nit fur euch were, und obgleich pfab seinen schwanz fur sich selbs gegen eu nit auspreitet, so nö̈chte er eu dannocht zuschub thun, die eu und dem handel und dem wesen zu Nurmberg untreglich weren. Wa ir eu dann an ihn wolltend richten, so trostet er sich grosser hilf. So wiste er, der waldfogel, das ime der Hess het zusagen getan mit leib und gut wider eu zu hilfen..... Ein hochverständiger Fürst, der von dem Versprechen der hessischen Hilfeleistung Kenntniss hat? Das sollte doch wohl der Kurfürst Friedrich der Weise von Sachsen sein, der mit Brandenburg und Hessen in Erbeinung und Erbverbrüderung stand. 1) Auch dass er sich nach dem oben erwähnten Bericht als Vermittler in den Streitigkeiten zwischen Nürnberg und dem Markgrafen anbietet, rechtfertigt die Vermuthung, ebenso wie die Notiz in dem Bericht vom 26. Mai, dass der $p \mathrm{f} a \mathrm{~b}$ sein gesicht gar scharpf hat gespitzt, als am herausgeen eichhorn, waldfogel und falk bei uns gestanden und gar gnedig zuǵesprochen haben. ${ }^{2}$ ) Als die Nürnberger aufgefordert werden, Schiedsrichter vorzuschlagen, rathen die Gesandten, Mainz, Kursachsen und Albrecht von Bayern dazu zu ernennen. Die Bezeichnung waldfogel kommt zum ersten Mal in dem Bericht vom 8. Mai vor und am 3. Mai ist der Kurfürst von Sachsen in Augsburg eingeritten. Am 25. April berichten die Gesandten, es gehe das Gerücht, Kurfürst Friedrich von Sachsen werde in den schwäbischen Bund eintreten, und am 1. Juli schreiben sie, die

1) Dase Nürnberg mit Friedrich dem Weisen gut stand, ergiebt sich aus den bei Müller Reichstagstheater unter Maximilian I. S. 727 angefuhrten Schriftstücken.

2) Das Verhältniss zwischen Friedrich dem Weisen und Markgraf Friedrich von Brandenburg war damals schon ein ziemlich gespanntes. So z. B. bat Letzterer am 25. Februar 1500 die Stadt Augsburg, ihm in den Streitigkeiten mit Sachsen Dr. Conrad Peutinger zu leihen und zuzuschicken. (Germ. Mus.). Am 6. April bittet der Markgraf Dr. Conrad Sterzel, ihm in den Streitigkeiten mit Sachsen zu rathen. (Berl. H. Arch.) 
Amsel halte gänzlich dafür, dass die waldvögel - dann man in handlung stund und des verzeichnus vor augen were - auch ir verstentnus mit den puntschen gewinnen. Gleich bei der ersten Erwähnung des Waldvogels wird bemerkt, dass sich Pfeffinger, des waldvogel s verwandter, gar gutwillig erzeigt habe. Dic Gesandten fragen deshalb an, ob sie im nun dic cisen mit etlichen negeln verheften sollen .... dann er bei dem waldvogel fur ander ist gelibt und gehort. 1) ...

Neben diesen weltlichen Fürsten stehen aber auf Seiten Nürnbergs ausser der Amsel noch andere geistliche Herren, die oben bereits als zur Rechten des Königs sitzend erwähnt wurden. Da ist zuerst das $\mathrm{Eich}$ horn. Auch diese Bezeichnung findet sich vor dem 3. Mai in keinem Bericht, und schon dadurch wird die Vermuthung unterstützt, dass darunter der Er $z b$ is ch of von Magdeburg, welcher zugleich mit dem sächsischen Kurfürsten auf dem Reichstage eintraf und sich ebenso günstig wie jener für Nürnbergr aussprach, zu verstehen sein wird. Dass mit der Bezeichnung Eichhorn ein höherer Kirthenfürst gemeint ist, ergiebt sich aus den Platze, den er einnimmt. Er kommit gleich hinter der Amsel. Und dass der Erzbischof von Magdeburg - damals ein sächsischer Prinz Ernst - nicht blos mit dem Waldvogel zusammen, wie oben bemerkt, den Nürnbergischen Gesandten gelegentlich beim Herausgehen gnädig zugesprochen hat, sondern auch mit der That behilflich gewesen ist, ergiebt sich aus einem Schreiben der Gesandtschaft an Bürgermeister und Rath zu Nürnberg vom 2. Juni, worin sie den Magdeburgischen Rath Dr. Preusser, der ihnen mannigfache Dienste geleistet habe, bei seiner Durchreise warm empfehlen. Magdeburg gehörte auch zu dem Ausschuss, der mit der gütlichen Beilegung der Zwistigkeiten betraut war. Andere Anhaltspunkte finden sich allerdings zur Beantwortung dieser Frage nicht. - Neben dem Eichhorn sitzt der Sal amander. Bei diesem ist dadurch ein kleiner Fingerzeig gegeben, dass in dem Bericht vom 13. Mai erwähnt wird,

1) Ein Bericht Nürnbergischer Gesandten vom 7. Mai 1502 liefert noch eine neue Bestătigung. Da heisst es : wir hören gern, dass der tag zwischen den vogeln nit furgang gewinnt, und halten es dafur, das der abschlag aus der ursach sei bescheen, das des aurhans jung, waltfogel und des pfaben bruder und der pfab am erchtag in den pfingstfeirtagen zu Erfurt zesamen komen. Diese Notiz bezieht sich unzweifelhaft auf die Zusammenkunft der brandenburgischen und sulchsischen Fürsten, die in Erfurt 1502 stattfand. 
dic Ciesandten hätten mit dem Salamander wegen Hans Rieters A rmenleuten verhandelt und ihm einen Brief gezeigt. Dieser Brief, dessen Abschrift beiliegt, ist an den Bisch of ron Würzburg gerichtet und auf diesen scheinen alle Beziehungen, in denen der S a l a m a nder auftritt, zu passen. An J. Mai wird berichtet, S a lamander sei bei zwei Tagen in seinem Nest gewesen und fühle sich schwach; er werde vielleicht nicht nach Augsburg kommen. Am 13. Mai aber erklären die Gesandten, sie seien u. a. auch beim s a a m and or gewesen; sie haben jene oben erwähnte Verhandlung gepflogen; sie hätten ihn noch nicht vernagelt, weil sein Junger nicht anwesend sei, der bei der Sache gut wäre, und aus Furcht, es sei nach Gelegenheit seiner früheren Forderung zu wenig. Nun war der damalige Bischof von Würzburg Lorenz (1495-1519) aus dem Hause Bibra; unter seinem Jungen 1 ) wird $H$ ans von Bibra, den die Gesandten schon früher in ihrem Bericht vom 9. April erwähnt hatten, zu verstehen sein. Am 26. Mai wird Salam ander unter denjenigen genannt, welche dem Könige zur Rechten sitzen; es sind dies lauter Bischöfe (vgl. Müller Reichstagsth. unter Fr. III. 6. Vorst. S. 7), und es könnte demnach höchstens der Zweifel entstehen, ob unter dem Sal a mander nicht ein anderer, in diese Verhandlungen eingreifender Bischof, etwa der von Worms oder der von Eichstätt, zu verstehen sei. Dagegen spricht nun aber entschieden eine Stelle in dem Bericht vom 8. Mai 1502, wo es heisst: Item, die amsel hat auch verlauten lassen: sie wiss, dass des pfaben bruder 2) dem salamander werde feld zuschieben, das er beschedigt werd; das habe des pfaben bruder ir, der amsel, selbs gesagt; desgleich werde der pfab stiglitz und haselhon auch thun. Dadurch werden die beiden Bischöfe von Worms ${ }^{3}$ ) und Eichstädt ausgeschlossen, da ihre Gebiete doch zu entfernt lagen. Eher würde diese letztere Notiz auf den Bamberger Bischof passen; doch gegen

1) Der Junge bedeutet allgemein: Anhänger, Rath oder Gesandter, nicht etwa naher Blutsverwandter.

2) Des pfaben bruder kann, da Markgraf Friedrichs leibliche• Bruder, Kurfürst Johann Cicero und Margraf Sigismund, schon seit 1499 bez. seit 1495 todt waren, nur Kurfürst Joachim I von Brandenburg sein. Er hatte schon 1500 entschieden auf Seite seines Oheims gegen Nürnberg gestanden. Dass Joachim als Bruder Friedrichs bezeichnet wird, ist nichts Seltenes.

s) An diesen könnte auch schon deswegen nicht gedacht werden, weil sich aus dem Bericht vom 8. Mai 1502 ergiebt, dass Salamander in der Nähe Nürnbergs begütert und mit der Reichsstadt im Bunde gewesen sein muss. 
diesen spricht wieder der Umstand, dass Sal a mander den Nürnbergern persönlich sein Wohlwollen auf dem Reichstage bewiesen hat, während die Anwesenheit des Bischofs von Bamberg in keinem dieser Berichte erwähnt wird. 1) Freilich müssen stiglitz und haselhon auch benachbarte Bischöfe sein. Das $\mathrm{H}$ a selh uh n sitzt am 26. Mai neben dem Salamander auf der Seite der Bischöfe. Ebenso wird er mit Salamander zugleich an 13. Mai als ein Freund Nïrnhergs erwähnt. Schon am 8. Mai wird er von den Gesandten um Hilfe: angegangen. Es bleibt kaum eine andere Möglichkeit, da 1502 das Haselhuhn neben dem Stieglitz vom Markgrafen bedroht wird, als den Bischof von Eichstädt, Gabriel von Eyb, darunter zu verstehen. 2) Dann würde als Stieglitz der Bisch of von Ba mberg übrig bleiben. In den Berichten aus dem Jahre 1500 wird er, so weit ich es verfolgen kann, nur zweimal erwähnt. Daraus lässt sich keine weitere Bestimmung gewinnen. Dagegen würde die Erwähnung des Stieglitz in dem Berichte rom 7. Mai 1502 (das hätte sie, die amsel, allein darum gesungen, dem dannocht nachzugedenken; man mochte dannocht je versteen, was uf die letz aus disem allem mocht erfolgen, damit ein grossern schaden zufurkomen und $d a m i t$ den stiglitz mit seiner richtigung auch angezogen etc.) auf den Bamberger Bischof wohl passen.

Sehr häufig wird auf Nürnbergs Seite das Keislin erwähnt. Aber eine Andeutung darüber, wer darunter zu verstehen sei, findet sich erst in der Relation vom 4. Juni. Da heisst es am Schlusse: der Kammermeister Maximilians habe sich nächten erboten, heut gute Botschaft zu bringen. In einem beigelegten Zettel wird erwähnt: das Zeislein sei wirklich gekommen, zuerst offiziell mit Dr. Neydecker als königlicher Bote, um einen Ausgleichsvorschlag zu überbringen; dann aber sei. er noch einmal allein erschienen u. s. w. Man wird diese beiden Notizen wohl mit einander in Verbindung bringen und demnach unter dem Zeislein den Kammermeister Maxiniliaus

1) Auch in der Ordnung des Reichsregiments (bei Müller Reichstagsstaat unter Maximilian S. 28 ff.) werden die Bischöfe von Würzburg, Eichstadt, Augsburg $u$ s. w., aber nicht der Bischof von Bamberg als anwesend unter den Zeugen angeführt.

2) Dass dieser vielfach in dieser Streitsache thätig gewesen ist, \%. B. als königlicher Kommissarius wegen der That zu Ried, ergiebt sich aus früberen Berichten der Nümberger Gesandten (März 1500). - Im November 1500 besuchte er Nurnberg. D. Stchron. XI. 594. 
W. Wolff verstehen dürfen. Dass dieser eine bedeutende Rolle bei den Verhandlungen gespielt hat, ergiebt sich aus einer Reihe von Notizen. So schreiben z. B. am 12. April die Nürnbergischen Gesandten: Auch geen wir dés chamermaisters hie irr; hoffen, wa wir den gehabt hetten, unser sachen solten numer anders steen. Darumb ist unser gutbedunken, das e. w. wolle furdern, damit er mit dem ersten her gen Augspurg kome, soverr das mit fugen kann gescheen. Diese Bitte muss erfïlt worden sein; denn schon am 23. April wirl berichtet, dass der König beim Verhör der Parteien ausser den andern Räthen auch den Hofkammermeister zugezogen habe. Am 25. April wird das Zeislein zum ersten Mal erwähnt; natürlich auch erst, nachdem der Bitte um das vocabulari gewillfahrt worden war. In diesem Bericht wird über eine Unterredung mit dem Erzbischof von Mainz berichtet; da heisst es: und wa es sie, die amsel, het gut bedunkt, so möcht ich, Anthoni Tetzel, wol leiden, das etlicher person, die am nechsten zu der handlung weren gezogen worden, verschont und das zeisle an die stat gezogen wurde.. . . . Heut ist das zeislin zu uns geflogen und hat uns gesungen, das es der sperber zu uns het gesandt, uns zu eroffnen, das es, das zeislin, und der ain des pfaben verwandten in gelben sporen, dem zeislin wolbekannt, von den geprechen lang, auch vil und mancherlai hetten geredt und sich mitainander entschlossen, in den schaden gutlich ze handeln, soverr sio das bei den tailen und zuvoran bei dem sperben mechten erlangen. Darauf habe Sperber und Pfau zugestimmt und so seien amsel, auerhahn, zeislin und dessen Bekannter mit der Verhandlung betraut worden. Am 6. Mai schicken die Gesandten auf Empfehlung des Kammermeisters (dem canermeister zu gefallen) den Georg Dickhaut zur Aufnahme in den Stadtsold an Bürgermeister und Rath von Nürnberg. In einem Separatbriefe schreibt Anthoni Tucher an 13. Mai an die Elteren, Maximilian habe vertraulich mit ihm geredet und erklärt, der Ueberfall markgräflicher Reisigen, geschehen zu Ried, sei eine schlimme That gewesen; doch sei er, der König, nichtsdestoweniger der Stadt Nürnberg in Gnaden geneigt und sehe es gern, wenn sie mit frumen, treuen leuten versehen sei; ebenso habe er die Verpflichtung, seine Diener, die from und erber weren, unterzubringen; kurz, er habe verlangt, sie sollten seinen Kammermeister W.1) Wolff

1) Der Kammermeister war ein Narnberger. Kind und hiess mit seinem 
zum Schultheissen von Nürnberg machen. Er werde ihn in die Aemter Werd und Weissenburg einsetzen; das sei für Nürnberg auch nützlich. Tetzel möge dieses Ersuchen ganz insgeheim dem Rathe der Stadt vortragen; ausser dem Herzog Friedrich von Sachsen wisse noch niemand von der Sache. - Am 26. Mai werden die Hofräthe Maximilians, die an den Reichtagsverhandlungen Theil nehnen, aufgezäblt: Eitelfritz von Zollern, Graf Wolfgang von Fürstenberg, Michel von Wolkenstein, Paul von Lichtenstein, Zeislin, Serentiner und Mathias Lang. - Am 28. Mai berichten die Gesandten, sie hätten ihre Vertheidigungsschrift dem Sperber durch das Yeislin übergeben. - An 7. Juni wird gemeldet, Maximilian habe Berthold von Mainz, den Kurfürsten von Sachsen, den Erzbischof von Magdeburg, Herzog Albrecht ron Bayern und die Bischöfe von Würzburg, Eichstädt und Augsburg zu sich gefordert und mit ihnen über die Streitigkeiten zwischen Brandenburg und Nürnberg verhandelt. Ihnen, den Gesandten, habe das Zeislein den Beschluss dieses Ausschusses mitgetheilt. Maximilian habe erklärt, die errichteten Thürme bis zum Austrage der Sache in seine Ver-

Volnamen Balthasar. Die Verwechslung der Buchstaben $B$ und $W$ ist damals nichts Seltenes. - Uebrigens erklart sich seine den Nürnbergern so wohlwollende Haltung zum Theil wohl auch daraus, dass Margraf Friedrich im September 1499 trotz der Fürbitte des Königs Maximilian dem obersten Schatzmeister desselben Baltasar Wolf das Gesuch, in Farnbach ein neues Gebäude errichten zu dürfen, abgeschlagen hatte. Im Bamb. A. findet sich ein darauf bezigliches Gutachten der markgraflichen Räthe, in dem es heisst: je mer wir die sach bewegen, je mer wir sorgfeltigkait und beschwerd in handel finden; dieses gebeu wurd furgenomen in der besten e. g. wiltfur um Cadolzburg, do der recht und best lust des gejaids ist. Es ligt auch Cadolzburg e.g. haubtsloss vast nahend und Nurnberg, mit den ir vil anstoss und irrung habt und euch gein ine teglich aufrur versehen must, nit fern und in der mass, das von dem kirchthurm zu Farnbach mag gesehen werden fur die thor zu Cadolzburg und Nurnberg, was da aus und ein kome . . . . . Darum konnen wir nit raten, das e. f. g. bewillig oder zulass an dem ort einich neu gebeu anders dann ain zimblich lustbaus on brucklich bevestigung zu machen. Was wolt die ro.ko. mt. e. g. zeihen euch damit zu beschwern, eurer eltern sel. ged. und e. g. selbst getreue dienste (mer dann eur und der euren vermugen ist) unangesehen, die billich gnad und belonung auf ine tragen und das s. ko. unt. euch hoher ansehe, dann s. g. schatzmeister, den Wolf, den s. ko. gn. wol in ander weg zu begnaden und zu erhohen hat . . . . . Später allerdings gestaltete sich das Verhältniss zwischen Markgraf Friedrich und dem kais. Rath und Kammermeister Balthasar Wolf von Wolfstall freundlicher, wie ein Brief Maximilians an den Markgrafen vom 15. Juli 1508 (Nürnb. A.) beweist. 
wahrung nehmen zu wollen. 'Zwar hätten ihm die Fürsten abgerathen, dies zu thun; aber der König habe erklärt, er wolle es ohne Schaden und Präjudiz thun. Zeislein habe sich angemasst, dies abzuwenden. - Nicht ohne Wichtigkeit für die Entscheidung dieser Frage ist noch eine Stelle in der Relation rom 18. Juni. Da erzählen die Gesandten (Dr. Johann Letscher, Anthoni Tetzel, Heinrich Wolff, Conrad Imhof), dass ihnen Maximilian den versprochenen Abschied nicht gewährt habe; sie seien in Zeisleins Gemach beschieden worden und von dort sei blos Heinrich Wolff zum König geführt worden. Dieser Heinrich Wolff war nämlich der Vater des Kammermeisters Balthasar Wolff. 1)

Weit weniger oft als das Zeislein wird die Kohlmeise von den Gesandten geuannt. Aber auch diese Persönlichkeit kann schwerlich zu den Fürsten oder Reichsständen gehört haben, sondern muss wohl unter dem Personal der königlichen Kanzlei gesucht werden. Zu dieser Vermuthung führt folgende Stelle des Berichts vom 1. Juli: die amsel ist auch ab dem unsteten wesen ganz ungeduldig, kann uns doch von der instruction, darauf die potschaft solle gefertigt werden, kein eigentlich wissen sagen, wiewol sie mitsampt der kolmeisen dieselben instruction mer denn einmal verzeichent het. . . . A Auch sonst steht die Kohlmeise mit dem Erzbischof Berthold in Verbindung. Am 25. Juni wird berichtet: die Kohlmeise habe den Gesandten auf Befehl der Amsel eröffnet, dass der pfab vor der versamlung, der Behem halben, die in mit macht sollen uberziehen, so statlich anregen hat gethan, das ihm anheims zu reiten ist erlaubt. ..... Auch in dem Bericht vom 18. Juni erwähnen die Gesandten, dass sie in einer Audienz der Amsel mitgetheilt hätten, die neue practica, die uf der pan sei, wüssten sie durch die Kohlmeise. Aus dieser Verbindung mit der königlichen Kanzlei und dem Erzbischof von Mainz möchte ich folgern, dass unter der Kohlmeise der Sekretarius Maximilians, Sixtus Olhafen, zu verstehen sei. Ehe das vocabulari eingetroffen war, erwähuen die Gesandten in dem Schreiben vom 7. April: sie seien in grosser Verlegenheit, da Dr. Joh. Letscher und der Rathschreiber Mulbeck aufgefordert worden seien, sich zur Haft wieder in Nördlingen zu stellen. Sixt Olhafen meine freilich, sie könnten ebenso gut in

1) S. Deutsche Stadtechr. XI. S. 615 A.1. - Dass Balth. Wolff auch sonst im Interesse Nürnbergs thätig war, ergiebt sich aus XI. 610. Ann. 7. 
Augsburg bleiben und habe versprochen, beim Erzbischof von Mainz darauf hinzuarbeiten. - Am 9. April wird geschrieben: der Erzbischof Berthold habe sich sehr gnädig bewiesen, ist villeicht gemaint, das e. w. nit pessern rucken mochten gehaben dann den pund, wiewol s. g. das mit kainem wort hat gemelt; desgleichen ist uns von ... herzog Albrechten in Pairn auch vil anzaigens getan und das e. w. mit ainer weis und mass darein komen möchte und solchs villeicht an eu gesinnen wurde etc. So hat Sixt Olhafen von derselben und andern sachen allerlai vertreulicher handlung getan und helt sich fast getreu und wol. ... In dem Bericht vom 15. April heisst es: Item des ba u m s halb ich, Anthoni Tetzel, mit Sixten geredt, der furter mit Mentz auch hat davon handlung gethan; des gnad sich hat verfangen in den feyertagen mit mir davon zu reden .... In dem Bericht rom 25. April ist noch einmal von Sixt die Rede: die Amsel versehe sich, dass Nürnberg in den Baum, d. $h$. den schwäbischen Bund, zu kommen versuchen werde, wie nun die ordnung und mass und sunderhait verlaut, ist dem Sixten bevolhen dieselben ding zu weg zu suchen.... Dagegen wird - zum ersten Mal in dem Bericht vom 5. Mai der Kohlmeise Erwähnung gethan. Amsel und Kohlmeise hätten den Bescheid, wie er in Maximilians Kanzlei begriffen worden sei, verbessert ${ }^{1}$ ) und ibnen, den Gesandten, gerathen, sich bittweise an den König zu wenden, damit die Gefangenen zunächst aus der Haft entlassen würden. - Beim Reichsregiment unterzeichnete (z. B. 12. Dezember 1500) Berthold: per regem B. archiepiscopus Mogunt. sst. Und andrerseis: In consilio imperii Sixt Olhafen Secret.

Damit ist die Reihe derjenigen Personen, die auf Seiten Nürn-

1) Sehr gut passt dazu die Bemerkung in den Deutschen Städtechron. XI. 630. Anm. 2: "Sixt Oelhafen stand schon unter Friedrich III. in kaiserlichen Diensten ..... Er war auch jetzt noch (1501) königlicher Sekretair, zugleich aber ${ }_{n}$ in der römischen cantzlei bei unserm Herrn von Majntz als römischem des reichs ertzcantzler" (aus dem Nürnb. Rechnungsbuch no. $24 \mathrm{Bl}$. 145) thătig." Er war fruher Karthäusermönch. Vgl. über ihn auch D. Stchron. XI. 607. -Er war auch 1502 im Interesse Nürnbergs thätig, wie einige Briefe im Nürnb. A. beweisen. Als die Gesandten der Stadt Mandate Maximilians gegen die grafen, herren und die von der ritterschaft im lande zu Francken, die verainigung mit einander zu baben anziehen, sowie gegen die Gebrüder von Luchau zu Gunsten Nürnbergs ausgebracht hatten, wurden diese nicht gentigend befunden und mit Verbesseruug von Sixt an den königlichen Hof zurückgeschickt, und wirklich erlangten die Gesandten darauf das Gewünschte. 
bergs stehen, erschöpft. Denn der Auerhahn, der nur in einer einzigen Relation (aus dem J. 1500 wenigstens) erwähnt wird, scheint zur Gegenpartei zu gehören. In dem Bericht vom 25. April heisst es: die amsel hat nir angezaigt, heut weren des pfaben canzler und noch zwen des pfaben verwandten in gelben sporen bei ir, der amsel, mer dann ainest gewesen und angehalten uf mainung sich in die handlung ze schlagen. Darauf ansel abschlegig antwurt het gegeben, der mainung, sie wisten, das dergleichen an sie, die amsel, vormals ron wegen des pfaben auch gelangt und das sie sich mit sampt der a urbannen und andern vor etlichen tagen gutlicher handlung bet verfangen. ... Nun ergiebt sich aus den vorhergehenden Aktenstücken üher derartige Verhandlungen nur Folgendes: Am 14. April berichten die Nürnbergischen Gesandten darüber, dass Sigmund Pflug, der Kanzler des Herzogs Albrecht des Beherzten von Sachsen, sich ihnen gegenüber zur Vermittlung erboten babe; ${ }^{1)}$ er habe ihnen erklärt, er sei von den markgräflichen Räthen zu gütlicher Handlung ermächtigt. - Ferner erzählen sie, dass sie vom Erzbischof Berthold erfahren hätten, Pflug habe sich auch an diesen wegen Vermittlung gewendet; derselbe habe jedoch darauf hingewiesen, dass man nicht wohl in Maximilians Vermittlung sich einmischen dürfe. Darauf wird am 15. April berichtet, dass vom Könige zu gütlicher Verhandlung neben dem Erzbischof von Mainz Herzog Albrecht von Sachsen und neben Eitelfriedrich von Zollern, dem Bischof von Triest und Dr. Neidecker der sächsische Kanzler Sigmund Pflug berufen worden sei. - Jedenfalls ist also unter dem Auerhahn der Herzog Albrecht von Sachsen zu verstehen. Auch bei einer späteren gütlichen Verhandlung betheiligt sich auf Befehl des Königs laut des schon erwähnten Berichtes vom 25. April neben der Amsel von Fürsten nur der Auerhahn. - Diese Vermuthung wird unterstützt durch den Umstand, dass aus den Relationen der Herzog Albrecht ron Sachsen, der übrigens noch in demselben Jahre 1500 starb, und der Auerhahn gleichzeitig verschwinden. Vom 25. April an geschieht ihrer keine Erwähnung mehr. Erst im J. 1502 finde ich im Bericht vom 7. Mai wieder die schon oben erwähnte Notiz, dass des aurhans jung waldfogel und des pfaben bruder und

1) Diese Bereitwilligkeit erinnert an Dietrich von Harras, der ja auch im Auftrage Albrechts von Sachsen 1496 zwischen Brandenburg und Nürnberg verhandelte. 
der pfab am erchtag in den pfingstfeirtagen [17. Mai] zu Erfurt zesamen komen. Ein sächsischer Fürst ist also unzweifelhaft unter der Bezeichnung Auerhahn zu suchen:1) wenn die oben gegebene Deutung des Waldvogels richtig ist, bleibt nur Albrecht und als .,sein jung" sein Soln Georg übrig.

Ausser dem Auerhahn gehört zur Partei des Markgrafen Friedrich von Brandenburg - denn Herzog Albrecht von Sachsen tritt allerdings nach den vorliegenden Berichten an 22. April entschieden für Brandenburg ein - der auch nur selten erwähnte Blaufuss (plofuss), welcher erst seit dem 20. Juni (wenigstens durch seinen Gesandten) in die Verhandlungen eintritt, obwohl er schon früher einmal gelegentlich erwähnt worden ist. Im Bericht von diesem Tage heisst es ausdrücklich, der Blaufuss äussere sich auch feindselig gegen Nürnberg. In der folgenden Relation vom 22. Juni rathen die Gesandten den städtischen Behörden nachgiebig aufzutreten. Denn einmal sei zu bedenken, dass sie sonst in den schwäbischen Bund nicht aufgenommen werden möchten, sodann aber, dass die Bündnisse mit den bayerischen Fürsten bald abliefer. Nach dieser Zeit hätten sie gegen Brandenburg nur Bamberg noch als Bundesgenossen, gegen den Blaufuss aber niemanden. Am 1. Juli heisst es: die Amsel rathe getreulich, das e. w. gedecht aus der sach zu komen, ob das gleich mit eim leidenlichen schaden geschehe, und den ruck darnach an ein ander ort zu lainen, wie dann des paums halbeu, als wir westen, auf der pan were; vermeinet auch, das e.w. alsdann bei ru und gutem frid wurde pleiben, obgleich plaufuss und pfab die kopf wurden zusammenstossen, als zu besorgen ist; dann des plaufuss geschickter hat sich hie bei dem ausschuss offentlich horen lassen, plauf u s s werde seine cloen (Klauen) in die thürnlin, die uf dem seinen steen sollen, auch schlaben, hab nur ein aufsehen, wie es dem pfaben wolle ausgeen etc. Schon aus diesem Passus geht hervor, dass unter dem Blaufuss ein dem schwäbischen Bunde angehöriger, eng mit dem brandenburgischen Markgrafen verbündeter Fürst zu verstehen sein wird. Jeden

1) Sollte der Einwand erhoben werden, dass in Erfurt auch die hessischen Fürsten als Bundesverwandte vertreten waren, so würde darauf aufmerksam zu machen sein, dass der Auerhahn genannte Fürst auf dem Augsburger Reichstage anwesend gewesen sein muss. Von einem hessischen Fürsten vermöchte ich dies nicht nachzuweisen. Am 7. Juni wird ausdrúcklich erwähnt, dass von Hessen nur Räthe anwesend seien. 
Zweifel zu heben ist aber folgende Stelle geeignet. Im Bericht vom 22. Mai besprechen die Gesandten die ihnen (freilich nur im hüchsten V'ertrauen) mitgetheilten Statuten des schwäbischen Bundes. Da heisst es nun u. a.: Zum vierden haben wir auch etwas beschwerden gehabt aus ursachen, die e. w. wissen zu bedenken, aus dem artikel, der sich anhebt: „Item so sein herzog Ulrich von Wirtemberg" im abschid zu Eslingen 1) bei dem zaichen $\square^{4}$; und die ansel auch darinnen ersucht und finden, das es neur ain schain sei; wa es aber zu schulden langet, das dieselben zwen wider den plofuss genaigter wurden sein $z u$ thun dann jemand andern. . . Daraus ergiebt sich, dass unter dem Blaufuss der Herzog Ulrich von Württemberg $\mathrm{zu}$ verstehen ist.

Es sind nun aber doch noch einige Schwierigkeiten zu beseitigen, die freilich nur eine untergeordnete Bedeutung für das Verständniss dieser Berichte haben. Am 25. April heisst es: desselben freitags und auch heut weren des pfaben canzler und noch zwen des pfaben verwandten in gelben sporen bei ir, der amsel, mer dann ainest gewesen etc. In derselben Relation wird noch einmal ain des paben verwandter in gelben sporen erwähnt. Am 8. Mai schreiben die Gesandten, der pfab habe den Mann in goldnen sporen, der dem Zeislein bekannt sei, auf der Jagd bei dem Sperber gehabt. Einen Anhalt für die Feststellung dieser Persönlichkeiten, die natürlich zu dem Gefolge des Markgrafen gehören, bietet der Bericht vom 15. April, in welchen als Räthe, welche die brandenburgische Sache führen, angegeben werden: der Marschall Hans von Seckendorf, der Amtmann zu Schwabach Veit von Lentersheim, der württembergische Kanzler Dr. Lamparter und Dr. Valentin ron Türkheim. Vielleicht, dass also der Ausdruck Verwandte in gelben Sporen sich auf die beiden ritterlichen Räthe und im Einzelnen der Mann in goldnen Sporen sich auf Veit von Lentersheim, welcher an königlichen Hofe eine angesehene Stellung einnahm, bezieht. Möglicher Weise geht anf ihn auch folgende Stelle in dem Boricht vom 7. Mai 1502: als wir diesen brief haben beschlossen, ist von der amsel ain schall irs lieblichen gesangs an mich, Anthoni Tetzel, gelangt, wie des pfaben jungen, ${ }^{2}$ ) die verstendig und frum

1) Dieser Artikel ist zu finden bei Datt de pace imperii publica p. 371 § 28 .

3) Wie oben schon erwahnt, bedeuten die "Jungen" die Räthe oder Gesandten eines Fürsten. 
seien, hetten sich in der handlung einer geschickten weis gehalten, aber etlich ander sein junge als clein Veitlein, der man mit den gelben sporen, der ein guten landsknecht gebe, und andér dergleiche mer, die weren gar einer ungeschickten weis und nit zu gutem geneigt. -

Schliesslich wäre noch eine Erklürung zı versuchen für den Ausdruck „die rom schwarzen erdreich". Er kommt in dem Bericht rom 22. Mai vor, wo es heisst: Darumb ..... mogen e. w. die sachen auch bewegen und uns eur gemut und mainung berichten; sein wir e. w. berelh mit allem fleis nachzefolgen willens und unrerdrossen, und wa dann e. $w$. hierinnen was gemaint sein will, das mögt ihr bei zeit furnemen. Daun der paum oder die fordersten este werden uf Johannis sunwenden schierstkunftig zesammen kommen; an demselben ort mocht man beschlieslich ron der sachen handeln. So mogen e. w. der vom schwarzen erdreich halben, ob und wie die auch hineinzupringen weren, nachgedenken haben. .... Es handelte sich also augenscheinlich um Verbündete Nürnbergs, denen gleichzeitig mit der freien Reichsstadt der Eintritt in den schwäbischen Bund ermöglicht werden sollte. Da nun ein Gesandter schon am 25. April berichtet: die amsel hat mir auch angezaigt, das die von Straşburg auch darein komen wurden und it\%o darumb hie seien und auch mit ainer mass, darzu wir dann mer raten und die von Windsheim als ander eur gewandten mit euch hineinzepringen aus ursachen, wa es zu schulden kome, das in nit besunder hilf wurde aufgelegt; dem waist e. w. wohl nachzugedenken und mit den von Windsheim davon ze handeln --. so liegt die Vermuthung sehr nahe, dass die Bürger ron Windsheim als "die vom schwarzen erdreich" bezeichnet werden.

II.

Bei anderen (aber aus derselben Zeit stammenden) Archivalien des Nürnberger Archivs fand ich später ein Verzeichniss, das auf den ersten Anblick als ein Schlüssel zu einer Geheimschrift erkanint werden konnte. Zuerst regte sich natürlich die Vermuthung, dass es ein für die oben erwähnten Relationen brauchbares Hilfsmittel sein werde. Ond in der That fanden sich einige Bezeichnungen, die zu den oben gefundenen Lösungen passten. So steht neben Keyser .. Sperber. Und wenn es in dem Bericht vom 7. Mai 1502 heisst: 
wiewol ron dem pfaben zuerst $100 \mathrm{karpfe} u$ sein gefordert und etlich mer $\mathrm{karpfen}$, die die amsel nit wollte melden, das were rom baum und etlichen esten gar fur schimpflich geacht und doch voll den 100 bis auf 50 , von 50 bis auf 25 komen, und wiewol die ansel und ander vogel den geschickten pfabischen sovil anzeigens gethan, das sie so vil $\mathrm{karpfen}$ solten fordern, were nit pillich. die samnung wurde das nit thun .... so ergiebt sich aus der Vergleichung mit den Verhandlungen des schwäbischen Bundes (bei Klüpfel Urkunden, u. s. w. I. S. 467), dass $100 \mathrm{Karpfen}$ 100000 Gulden bedeuten; ferner beweist der Inhalt, dass unter der samnung die Stadt Nürnberg zu verstehen sei. Für beides findet sich in dem vorliegenden Schlüssel eine Bestätigung; denn hinter Hundert Reinisch guldin steht . . Carpf - freilich bedeutet 1502 Karpfen 1000 Gulden - und auf Nürmberg die statt folgt .. Sampnung. - Bei näherem Zusehen erwies sich jene Hoffnung. die anderen Geheimausdrücke der oben besprochenen Berichte hier aufgelöst zu finden, doch als trügerisch. Denn Amsel wird in diesem Schlüssel gebraucht für den Grafen Ulrich von Oettingen, Haselhuhn für den Bischof von Köln, Blaufuss für den Herzog Ludwig .von Bayern, Falk für den Pfalzgrafen, Sittich für die Stadt Windsheim, Zeislein für den Ausdruck ,etlich stett an Uln hangende", Kohlmeise für Qln, Stieglitz für das Kloster zu Reun, Eichhorn für den Ausdruck „ein scheinbrief solcher schrift man sich halten soll," Ast für „einnemen und ausgeben" und an andrer Stelle für jar, Auerhahn für Quittantzen, Nagel für Wald das Schloss. Es bedarf keines Beweises, dass diese Lösungen auf die Relationen der Jahre 1500-1502 nicht passen - weder zu dem Inhalte, noch zu den Marginalbemerkungen. Folglich musste der Schlüssel in einem - anderen Zeitabschnitte - wohl in einem früheren, da von Herzog Ludwig von Bayern, der 1479 gestorben war, von Herzog Albrecht von Oesterreich, der 1463 mit Tode abging, von Herzog Friedrich von Sachsen, der 1464 das Zeitliche segnete, Albrecht Achilles von Brandenburg u. a. die Rede ist - gebraucht worden sein. Er besteht auch nicht blos aus geheimen Ausdrücken für einzelne Persönlichkeiten, wie sie in den oben besprochenen Relationen vorkommen, sondern er bietet das Arsenal zur Abfassung ganzer Berichte in Geheimschrift, giebt ganze Redensarten, für die als Geheimzeichen meist ein einziges Wort gewählt ist, und bandelt von den verschiedenartigsten Gegenständen. Unter diesen Umständen 
wäre an eine Entzifferung der mit diesen Mitteln geschriebeneu Dokumente wohl nicht zn denken, und insofern gewinnt dieser Schlüssel als die einzige Möglichkeit, Aktenstücke von gewiss hervorragender historischer Bedeutung verstehen zu lernen, eine erhöhte Bedeutung. Da er nun seinem jetzigen Lagerorte nach für Entzifferung derjenigen Stücke, auf die er passt, verloren wäre, so dürfte es angemessen sein, ihn für alle Fälle hier in extenso mitzutheilen und den Versuch zu machen, wenigstens ungefähr die Zeit seines Gebrauches zu bestimmen.

Vorher seien noch einige Bemerkungeu äusserlieher Art gestattet. Der Schlüssel besteht aus einem Schmalfolioheft von 20 Blättern, von denen aber nur 14 beschrieben, die letzten dagegen leer sind. In dem folgenden Abdruck sind die Seiten jeden Blattes mit $a$ und $b$ bezeichnet. Der bei weitem grösste Theil ist von einer geübten, gut leserlichen Kanzleihand, deren feste Schriftzüge den ältesten Lettern der Incunabeln sehr ähnlich sind, geschrieben. Sie kann der Mitte oder dem letzten Drittel des 15. Jahrhunderts angehören. Manche Umstände deuten darauf hin, dass der Schreiber nur eine Abschrift anzufertigen gehabt hat. Denn er hat mehrfach Zeilen, die er an unrichtiger Stelle hingeschrieben hatte, ausgestrichen und am richtigen Orte eingesetzt. Nicht immer hat er mit Aufmerksamkeit abgeschrieben und es haben sich deswegen Korrekturen von anderer Hand als nothwendig herausgestellt. Ein charakteristisches Beispiel findet sich auf fol. 4 a, wo er Eychelleut geschrieben hatte, so dass eine andere Hand erst Eygenleut zu verbessern hatte. Dieser Schreiber nun (wir nennen im Folgenden seine Schrift die erste Hand) hatte die Folioseiten so eingetheilt, dass auf die etwas grössere linke Hälfte diejenigen Ausdrücke, für welche Geheimwörter gebraucht werden sollten, eingetragen wurden; dahinter machte er stets zwei Punkte (und das gehört zu den charakteristischen Eigenschaften seiner Hand) und dann folgte erst das Geheimwort. So z. B. gleich im Anfang: Abbt zu Sant Egidien .. Abguck. Wenn er den eigentlichen Ausdruck nicht auf eine Zeile brachte, so deutete er durch einen nach rechts hin konvexen Winkel die Zusammengehörigkeit der Zeilen an. - Für jeden Buchstaben des Alphabets bestimmte er mindestens eine Seite, so dass auf fol. $10 \mathrm{~b}$ nur ein einziges Wort steht. Die einzige Ausnahme macht der Buchstabe $\mathrm{W}$, der mitten auf fol. $13 \mathrm{~b}$ beginnt. Die Schriftzüge dieser ersten Hand zeigen, weder was den Duktus noch lie T'inte anbetrifft. merkliche Unter- 
schiede, so dass dieser Grundstock (hier in der Abschrift wenigstens) in einem Zuge niedergeschrieben worden zu sein scheint. - Ob der Schreiber nun absichtlich oder blos zufällig Raurn zu Ergänzungen und Nachträgen gelassen hatte, jedenfalls finden sich fast auf jeder Seite Zusätze von anderer Hand. Gleich auf fol. 1 a stehen am unteren Rande der Seite zwei Zeilen von einer offenhar jüngeren sie wird im Folgenden als 4. Hand bezeichnet - flüchtigen, schwer leserlichen Hand, die nicht Punkte zur Trennung der eigentlichen Ausdrücke von den Geheimwörtern, sondern ein dem kleinen lateinischen $\mathbf{v}$ ähnliches Zeichen verwendet. Von ihr rühren übrigens nur wenige Einträge her. Diese finden sich nur auf den ersten Seiten und stehen, mit Ausnahme der zwei Zeilen auf fol. 1 a, die eben ganz unten am Rande hinzugeschrieben worden sind, hinter allen übrigen Zusätzen. - Etwas häufiger tritt eine schwerfällige, wenig im Schreiben geübte, aber doch sorgfältige Hand - nach unserer Bezeichnung die zweite - auf. Sie verbessert die Irrthümer des ersten Schreibers, fügt aber auch die meisten der vorhandenen Ergänzungen hinzu. Sie macht ganz den Eindruck, als gehöre sie einem Nürnberger Würdenträger an, für den der erste Schreiber die Abschrift dieses Schlüssels besorgt habe. - Schwer zu unterscheiden ist dann noch eine dieser zweiten ganz ähnliche, nur etwas aufrechter stehende Schrift, die wir einer dritten Hand zuschreiben, die aber möglicherweise auch von der zweiten herrühren könnte. Auch die Trennungszeichen dieser beiden Hände sind ziemlich gleichartig, bald einem nach unten spitzen Winkel, bald einem kurzen senkrechten Striche ähnlich. -

Wir lassen nun den Schlüssel selbst folgen.

(fol. 1 a. - Erste Hand:)

Abbt zu Sant Egidien .. Abguck

Abbt auff dem Munchberg .. Affeltar

Abbt in der Neuenstadt .. Coppaun wasser

Alt bischove zu Mentz .. Gans

Anlass .. Glas

Antreffent hilff oder kriegsleufft yztund vor augen $\}$. . Jeger

Ander eingelegt od. zugeschickt $\mid \because$ jung schrift.

Artigkel . . König von Aycheln

Anzusehen oder verwilligen .. kampff
Andree Barbirer .. Miltz

Artigkel der weysung . . Neujar

Abbt zu weissenburg am Rein ..

Ófenlin

b Auflag oder anstallung .. parisbiren Abrede oder langen .. pomerantzen Ablesen .. predigstul

Andree Scherlein .. prugau

Allen seinen willen erlangt . . Rottsittich

b Ausfuren .. Reytten

nō Anheben oder angefangen .. Retter

Anthoni paumgartner. (Er wird [deutache Stchr. X. 288] beim Jahre 
1464 erwähnt; doch gehörte er schon seit 1462 zu den jüngeren Bürgermeistern des Rathes. Vielleicht ist es derselbe, der beim Jahre 1452 [ebenda S. 197] neben den [ebenfalls hier genannten] Ulrich Arzt und Gossenbrot erwälnt wird) .. Spinnenwebe

Augspurg . . Troschel

Argkwon und verdechtnuss . Welischen wein

Auff das allerhertigst gehalten . . wildzeitig

Aller sachen und handlung . . weyss

Anlegung oder aufsatzung . . Agerlasterer

v Auff das böchst gepetten und ermant . . den himel zeygen

(Vierte Hand :)

Allen und yeden $\checkmark$ wisen

anwalt $u$ Vogler

(fol. 1 b. - Erste Hand:)

Abgesagt: brieff

Althern zu Nürmberg (Die 7 eltern hern. Vgl. D. Stchron. XI. 794). . Star Allen darzı verwandt . . IX hertzen Arbait . . X Schellen

Anschlag oder fürnemen . . ...

Anderthalb vorschub .. hofer (Ursprünglich stand hier dasWort hauren; das ist aber ausgestrichen $u$. yon andrer Hand hofer hingeschrieben)

Alle die disen kriegsleuffen verdacht weren Amerallen

E.ein ald urfechd. Bodenn. Alweck das Sloss (vielleicht das im Kriege Albrecht Achills gegen Herz. Ludwig d. R. 1462 ofter erwähnte Albeck. Vgl. Hasselholdt St. I 249) . . hawe

Alt hanns waltstromer (erwühnt in der Geschichte Peters von Argon [D. Stchron. V. 39j]. Er wird unter dem Buchstaben $H$ noch einmal erwähnt) . . kachel

Alt Spital . . frondinst

b Auff ainglube. Schachtel
(Zweite Hand:)

b Aufbot $V$ frisch heu

nó abslaen $\vee$ Gremen

nō abzuwechseln $V$ ansehen

Antwort geben $V$ pipin

nó Aufgelubde $V$ Sattel

Abscheide $V$ furreiten

Aufzeichen $V$ faden

Am lande zu Osterreich $V$ am taubenberg

(Dritte Hand:)

App.lliren | leuten

(Vierte Hand:)

Allen vleiss furkeren I all pfärd in pflug setzen

Anligend und Merklich beswürd | be dächtlichaitt

Achter und aberachter / gleich und ungleich der pflicht

Anhangk | hagken

Abentheur ! Nachpaurschafft

Abbt zu Kempten | Scheppler

Anth(oni) Tucher (S. D. Stchr. XI.

446 ff. + 1476) / Schanloch

Anth(oni) Holtzschuer (Burckhardt

d. funfft merckisch buech. 113) |

Smalholtz

(fol. 2a. - Erste Hand:)

Brieff . . Behmisch

Bestellung . . Beystadel

bischof zu freysing . . Citron

babst . . Fsch

banir des Reychs . . Erbotz

berchtold happ .. Eneas

bei oder ingegenwürttikait . . fürst

bischof zu Trier . . Gruntel

bischof zu Wirtzpurg. . Galander

bot .. hann

bischof zu Cöln . . haselhun

bischof zu Eistett . . henfling bei dem höchsten bepst- $\}$.. kue
lichen panne

büchsenmaister . . kneller

ber Cramer . . Maler (Sollte damit der Bakkalaureus Michael Kramer [D. Stchron. XI. 769] gemeint seiu 
und ist derselbe identisch mit dem (X.41 \&ff.] genannten Kanzleischreiber Michel Cromer, dem „Ausgeber" in burgundischen Kriege? Anfïnglich sollte dies Amt der auch hier vorkom mende Jorg Spengler übernehmen) bischof zu Agram . . Rotkoppff bischof zu Saltzburg . . Rotterthauber bischof zu Augspurg . . Rottamsel bischof zu Metz . . Reinswalb beslossen uder verslossen. . Rex berümen oder Geuden . . Rabin betschett . . Rosenwasser bischof zu Bamberg . . Sprintz (spriuz kleine Art Falken Cf. SchmellerFrommann II. 705. Dem Ausdruck Blaufuss verwandt)

burger . . Scheyt

bernheim . . Stockeul

burgkhausen . . Sara

bischof zu passau .. thauber

brieff zu halten. Reichstett. Y VogelStattsteur und Zins $\}$. hund

Berg . . Weyden

bestettigung . . Wasserrosen

bischof zu Gurck . . Zagelmayss (der Vogel parus caudatus [S. Schmeller-

Fr. II. 1089])

brannger . . Zucht

billich gehalten .. V hertzn

beswernus . Morgenschein

(fol. 2b)

böckler gesellschaft in Beyren .. pockstirn (Ueber die Abstellung dieses „Pöcklerbundes“ finden sich einige Notizen bei Krenner Landtagsh. [VI. 99])

Brief und sigel . . buchstab

Branegkisch . . Branber (Brauneckisch ? Albrecht Achill kaufte 1448 Brauneck vom Burggrafen Michael von Magdeburg, der weiter unten erwahnt wird. Vgl. auch Burkhardt d. funfft merkisch buech S. 182)

Besserung . . Obermain von Aicheln Bachenstein u sein helffer .. Wendung brandschatzung . . Weydenkoln
(Zweite Hand:)

Botschaft $V$ pautz

Berchtolt Thum $V$ panther

Bestellen V Creutz VIII

Blakeren Cancelley $V$ Sterisch Can celley (S. unten unter steyrich can(elley)

(Dritte Hand:)

Betzalung $\mid \ldots$.

Bekentnus Kennen

$$
\text { (fol. 3a. - Enste Hand:) }
$$

Clag oder ansprach . . Arbais (arbais $=$ Erbse)

Clagweyse . . Arbaisweyse

Cron zu Beheun . . Bernlinkrantz

Comiss oder gewalt . Comat (komat $=$ Kummet. $\quad$ S. Schmeller - Frommann I. 1246)

$\left.\begin{array}{l}\text { Coburgk und dieselb } \\ \text { gegent alle }\end{array}\right\}$. . Kieffarbais (Kief-arbais, die noch grüne Erbse.

S. Schmeller-Fr. I. 1229)

Creutzpredigen . . Karfunckel

Cardinal zu Augspurg . . Rottamsșell (jedenfalls der Biachof Peter von Schaumburg [1424-1469]).

Closter zu Reun . . Stiglitz (wohl identisch mit dem Orte Rain, wo Herzog Ludwig von Bayern im Dezember 1461 Urkunden ausstellte Vgl. Hasselholdt II. 580)

Collacionirt abschrifft . . Swartzpfert

Commission || Nussplatt

Commissari .. Nusspaum

Camer .. Taschen

\section{(Zweite Hand:)}

Copeyen $V$ umbslege

Cristoff frolich $V$ premer

Creutz .... V bestellen

Cristen $V$ vesten

$$
\text { (fol. } 3 \text { b. - Erste Hand:) }
$$

Dem allmechtigen Got $u$. seiner wirdigen mutter lob und dank
zu sagen (ainkürn = Einhorn) 
Die person, so auffsteen und ent-
rinnen

$\left.\begin{array}{l}\text { Der frefelhalb darüber die fünff } \\ \text { erkennen }\end{array}\right\}$. B (Die Fanf sind eine Nürnberger Gerichtsbehörde, die alle "Bchmachsachen und injurien " verrechten. $D$. Stchron. XI. 797.)

Daniel Ulmer .. Darmvol (erwähnt D. Stchron. V. 413)

Do das datum steet . . Geyss

Dinckelspühel . . Hering

Das wirdig hailigthumb. . Hertz (heilig tum = Reliquie spec. die Reichsheiligthümer. Vgl. D. Städtechron. XI. 839)

Deutschmeister . . Kressen

Doctor Rott .. Linckauth. (Ein Dr. Joh. Rot wird in Muffels Prozess [ also ca. 1468] erwähnt. Städtechroniken XI. 767.)

Das sich kranch wol mug der schrifft halten und gentzlich .. Ochs daran lassen

Die Stett Freyburg in Üchtland . . Pin. Bern Soliter Lucern stock

Doctor Kilian .. Ratgebe (wohl Dr Kilian von Bibra, Domherr zu Würzburg. Vgl. D. Stchron. X. 431 u. a)

Doctor Jopp ... Swalb. (Dr. Jopp 1461 erwähnt bei Hasselholdt-St. I. 187.)

Doctor Knorr .. Trapp. (Dr. Peter Knorr, Pfarrer bei St. Lorenz 1456, aber auch Anwalt Albrecht Achills $\dagger$ 1478. Vgl. Städtechroniken XI. 471.)

Den costen oder das gelt .. underman von clee

Diebstal . . vortail

Die drey obersten hauptleut.. Zellter. (Eine nürnbergische Behörde; vgl. D. Stchron. XI. 794. Allerdings könnten damit auch Reichshauptleute gemeint sein, deren es z. B. 1461 drei [Albrecht Achill, Ulrich v. Württemberg, Karl v. Baden] gab.)

Demuttiglich ersucht und \}.. hoppffgepetten
Doctor Jörg Heimburg .. hausen. (Es scheint damit der bekannte Dr. Gregor Heimburg gemeint zu sein. $\mathbf{Y g l}$ Deutsche Städtechroniken X. 26:3.)

Doctor Hanns birckheimer . . Josepp. (Hans Birckheimer wird als Raths freund erwähnt 1459. S. D. Stchron. X. 39j.)

Doctor Hayden .. Bollen

Dann wo zwen hungrig hund sein, do der ain isset und der Rott andere zusehen muss, tutt zu-
mal wee

Den nechsten weg oder strass .. Scher messer

\section{( $Z$ weite Hand :)}

Dinst $V$ springen

Doctor Schutz V Josephus

Doctor Seiffried $\vee$ Boech (erwahnt in Muffels Prozess XI. 765).

(Dritte Hand:)

dyenner | anstenner

$$
\text { (Andere [zweite?] Hand:) }
$$

Doctor pfeffer | premser (erwähnt $D$. Stchron. XI. j16).

$$
\text { (fol. 4a. - Erste Hand:) }
$$

$\left.\begin{array}{c}\text { Ein scheinbrief solcher schrift } \\ \text { man sich halten soll }\end{array}\right\}$. . Aichom Ein fride auff etlich jare .. Adam. appffel

Einung oder puntnus . . Armhaur

Ein nemlichen rechttag setzen . . Berund bestimmen niscliglas

Einnemen und ausgeben .. Ein Ast

Eylent .. holtzbirn

Ein eylent ganz frisch furnemen . . Jagd. der vermelten sach aller hund

Eingemengt . . Jatzinckten

Etlich stett an Augspurg und $\}$. . lerch
Ulm hangende Ernst oder herttikait .. Mirstattel

Erung oder schenk .. Walfisch

Eysenne püchsen .. Zwirle

Eyde oder pflicht .. Zwanck

Erfaren gewar wurden .. V schellen 
Erasem thorer .. Slech (jedenfalls der als bayerischer Kammermeister 1461 bei Hasselholdt-Stockheim II. $\mathbf{0} 99$ er wähnte Asm. Torr. - I, 247 heisst er auch Erasmus Torrer).

Erlangt oder erraicht . . III hertzen Erbvörster .. Umbgener

Erstorrt und verderbt worden . . lux

Essling . . henn

Etlich sachen etwas zugericht . krenchin

Erlangt sachen . . Mistler

Echter und aberechter . . pflicht

Etlich Stett an Augspurg hangend .. Swan

Erben . . Slang

Frschreckt . . Saffir

Eygenleut . . Tanzappffen. (Ursprüng lich stand Eychelleut da; die zweite Hand hat das Wort enst verbessert in Eygenleut.)

Erbhuldung .. Undermann von hertzen

Etlich Stett an Ulm hangende . . Zeislein

Ersprossen oder gefurdert . . :::

(Dritte Hand :)

Echter und aberechter $V$ gleich und ungleich oder pflicht

Elyas oder .....V herr weihartt (s. unten bei dem Buchstaben W)

$$
\text { (fol. 4b. - Erste Hand:) }
$$

Fride . Andacht

feuchtwang . . Abbtey

freyheit . gnadenjar

fussvolk . . flemming

feler . . jarhant

franckfurt ... krebs

Frantz Waltstromer . . kechelein. (Ein Franz Waldstromer wird in der Geschichte Peters von Argon erwähnt. D. Stchron. V. 395.)

furstenthum .. Neste

freund .. Nachpar

freyenstatt . . Oberbank

fochburgk . . Badschefflein

flucht . . Stockzaun fürzuhalten .. VII Aichelen

frey . . gedult

fürnemen oder handeln . . dinckel

fürsten . . fürwitz

fischgall am kaiserlichen hof .. Rumpf

(Fiscal)

freystett . . undermann von Schellen

freund oder furderer. . II Aichelen

(Zweite Hand:)

falscheid gesworn $V$ futerswingen

f.. dling $V$ frevel

$$
\text { (fol. } 5 \text { a. - Erste Hand:) }
$$

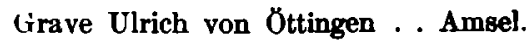
(Von 1429-1477. Vielfach erwahnt bei Hasselholdt-Stockheim I. 195 ff.) Gesworn eyd und pflicht .. Antlätz Grave Rudolff von Sultz .. Alber. (Rudolf IV. kaiserlicher Rath, Hasselholdt-Stockheim I. 335.)

Grave Hans von Wertheim . . Biber. (Johann III. 1454 - 1497. Erwähnt im J. 1462 bei Hasselholdt-Stockheim II. 609.)

Gewalt oder hannden . . Balast

Grosslüg oder unwarheit . . fuchs

Gefangen . . friesen

Gretz .. Gegler

Geest, getrenckt und gesterckt . . gehelligt

Geverlich listikait . . Grülaub

Geteget . . genettigt

Gelt .. gertten

Grave Haug von werdenberg .. Gunther von werd (er wirkte von $1460-1508$ )

Gnedigen hern .. Granhennffling

Geantwort . . geetzet

Grave Michel von Maidburgk .. Grasmair. (Wohl Burggraf Michael von Magdeburg, Graf zu Hardeck. Vgl. Deutsche Stadtechr. III. S. 397, 399; kaiserlicher Hofrichter. Siehe auch Stälin Wirt. Gesch. III. 474 und Siebenkees Materialien IV. 699.)

Gossenprot . . geysspart

Grave Hans von Eberstein . . Gauffer. (Graf Johann von Eberstein starb 
1479. Erwahnt D. Stchron. II. 449 im J. 1450)

Geruwet oder gefeyert . . hantzwehel

Gebotten . . heu

Gesellschaft sant Jorgen schilt . , hasel

Gutlich tage .. igel

Geschickt oder gesanndt als anwalt . imber

Gült oder Zinss . . jamahwe

Gross verdurplich scheden . . kersen

Gerechtikait . . kirchwey

Gabriel Tetzel . . liligenstengel ( $\mathrm{Vgl}$

D. Städtechr. X. 159 ff.)

Grave Eberhart von wirttemberg . . Neunaug (Graf Eberhard v. Würtem. berg 1457-96)

Grave Ulrich vonWirttemberg . Raiger (Graf Ulrich v. Württemberg 1433 bis 80)

Gefangen oder Niedergelegen .. Rüdenhunt

Gute richtignng und loblich ende .. Wasserbirn

Grave Schaffrid von leyningen . . Wes. pen. (Erwähnt 1463 bei Hasselholdt I. 272; 1471 beschenkt von den Nürnbergern. [Städtechr. XI. 516 Anm. 3.] Der bei Hasselholdt II. 94 erwähnte Graf Schaffrid von Hein gen ist in Leiningen zu verbessern. Vgl auch II. 103)

Gnediger herr . . Wilkür

Gehaust, gehoft, geetzt, getrenckt .. Wolff

Genomen oder abhendig gemacht . . Windenhund

$$
\text { (fol. } 5 \mathrm{~b} \text { :) }
$$

Genanten . . Zamerbere

Giengen .. Ziella

Gleit . . Zwirn

Getraut . . IX Aichelen

Gemach und nit girlich . . VII clee

Geraicht oder gelangen . . $\cdots$

Geschreckt . . Erper

Gütlikait . . Vinstern

guldin pull . . Otter
Glauben oder wathait . . Roth

Gemain reich und arm . wildper

Geheym oder verswigen . . Weissrosen

Gross .. VI von Schellen

$$
\text { (Zweite Haml:) }
$$

Gremssich V Greittich

Gefengnis $V$ Summerlaube

Gesieget $V$ bestympt

Gezeuge $V$ futer

Getreide $V$ zymmerwerk

Gehorsame underton $V$ regler

Grave Ott von Hennenberg $V$ habich

(Graf Otto von Henneberg 1465 bis 1502)

Geoffenet $\vee$ Tröt

$$
\text { (fol.6a. - Erste Hand:) }
$$

Hausfrau . . Amelich

Herm von München . . Begsteltz

Hans Mercklein . . Cleebat (Vgl. D Stiidtechr. Xl. 568 Anm. 3)

Hans Harscher wirtembergisch. . Dorn

(Erwähnt als Mitglied der württemh. Regentschaft 1468 bei Stälin III 553. S. auch Sattler III. 198)

Helling . . Ellsesser

Herrn und der Adel . . Finsidel

Hertzog Ernst zu Sachsen . f fasan (Herzog Frnst von Sachsen 1464 bis 8i. - Zuerst stand Friedrich da, wurde aber ausgestrichen und von anderer Hand Ernst darüber geschrieben. Uebrigens regierte dessen Vater Friedrich d. Sanftmüthige v. 1428-64).

Hans Gewolff . Geltnutz (Gewolf erwähnt als Hauptmann der Böcklergesellschaft bei Krenner VI. 102).

Heuptleut oder hanptman . . heuschreck

Hertzogen Aurach . Himelstatt

Helfer . . habern

Her hans von Fraunberg zu Prunn . . hausknecht (Rath der Herzöge Johann u. Sigismund von Bayern. S. Hasselholdt-Stockheim II. 599, erwahnt im J. 1461) 
Her Bernhart von Schoinherg . hecht

(Vgl. D. Stchr. III. 39i)

Hans kesicken . . Widerumb

Heyratgut . . hopfgart

Hertzog von Burgundi . . kraue

Hauffen . . knotten

Hennil . . kropen

Heinrich Span . . Murffeltier (erwännt bei Hasselholdt-Stockbeim II. 557,562 ) Her Jorg von Gich thumher
zu Wirtzpurg

(S. D. Städtechr. X. 431; Hasselholdt I. 101).

Her Wolfgang vorchtnauer .. Norff (S. D. Städtechr. X. 398 ff.; Hasselboldt-St. I. 241).

Hoheneck . . Niderberg

Herzog Albrecht von Oesterreich .. pluteisicken. (Jedenfalls ist Herzog Albrecht VI [der Verschwender] gemeint 1424-63)

Herzog Ludwig von Bayern . . plau. fuss (Herzog Ludwig von Bayern Landshut 1450-79)

Herzog Ludwig von Veldenz. . . pirickhan (Ludwig der Schwarze von Vel denz 1453-89).

Herzog Ott . . Rottauge (Herzog Otto v. Mosbach oder Neumarkt 1461-99).

Her Heinrich Marschalk. . Regelbirner (Vgl. D. Städtechr. X. 268).

Heintz Seybot. . Schnaugk (Als branden burg.Gesandter erwähnt bei Hasselh. St. I. 263).

Haims Orttolff .. Specht

Herzog Wilhelm von Sachsen.. Turtel taub Wilhelm III. von Sachsen 1428-82).

Herzog Sigmund von Oesterreich . . Tenlein (1439-96).

Heimlich sendbrief . . Tier

Hans Müllner . . Tunckel (S. D. Stchr. II. 396. X. 398. XI. 756).

Haidenheim . . Wasservalle (S. Hasselholdt I. 233).

Haidingsfelt . W Wiesel

Hardlung . . VIII hertzen
(Zweite Hand:)

here oder leger $V$ konig ron Schellen (fol. 6 b. - Erste Hand:)

Hoffnung . . IX schellen

Hörn oder vernemen .. V klee

Her Michel von Saunsheim . . hausvol

(S. D. Städtechr. II. 146).

Her Jorg von Stein .. Tann (S. D Städtechr. X. 278. - Hasselholdt Stockheim II. 374 ff.)

Hundert Reinisch guldin . . Carpf

Her Ulrich von Graveneck .. Abraumer (D. Stchron. V. 291. Anm. 3).

Her Jakob Rauscher pfarrer ... Jakob zu Weyssenburg samuel

Hertzog Victorin . . Ispen. (Dieser Sohn Georg Podiebrads regierte in Troppau von $1462-1500$. Er wird aber als Herzog auch schon 1461 bei Hassel. holdt-Stockhein II. 481 erwähnt).

Hengst und harnasch verlorn .. Gebrechen

Here oder leger . . küng von Schellen Hans Waltatromer alt . . kachel. (Hans Waldstromer erwähnt in der Geschichte Peters von Argon. D. Städto chron. V. 39j).

Heimlich gemech . . Nest

Her Cristof Morsperger . . Spieling

Hantschrift . . Turnay Ross

Her Wilhelm Trauner.. Weisstrapp

(Zweite Hand:)

herzog Ernst in Sachsen $V$ phassn (1464-86)

herzog Albrecht sein bruder\} $\vee$ Tobias (1464-1500)

herzog philips pfalzgrave $V$ hoenschein $(1476-1508)$

herzog Albrecht zu Munchen $V$ frischermut (1464-1468 regierte er zusammen mit seinen Bruddern; von 1468-1508 allein).

her Sigmund leiningen $V$ hopfgartner heinrich voyt marschalk $\vee$ platzmeister 


$$
\text { (fol. 7a. - Erste Hand :) }
$$

Interrogatoria . . Anclopfen

Jar . . Ast. (Oben bedeutete ast Ein. nehmen und Ausgeben.)

Jobst Tetzel . . Cromatfogel. (Vgl. D. Stadtechr. X. 216 ff.)

Juden . . Fledermaus

Jörg Spengler . Jaspis (Rathsschreiber 1488-91. Vorher D. Städtechr. X. 414 vorgeschlagen zum "Ausgeber"s) Johanns Muffel . . Stentz (Probst 2u St. Stephan in Bamberg, Sohn Nikolaus' M. D. Städtechr. X. 258. XI. 775) Jörg Spalter . . Spelten

Innhelt oder ausweyst . . Veyel

Jörg von Wendingen . . Wiltfang. (S. D. Stidtechr. II. 429. - Hassel holdt-Stockheim I. 145. - Hofler, Kais. Buch Albrecht Achills 8. 48 ff.) Johann Feucht .. widerumb. (Oben bedeutete widerumb Hans Kesicken. Hans Feucht ist Schreiber der Nurn. berger, bei der Rother Richtung 1460 . S. Hasselholdt I. 100)

Jobst Haller . . Wachelter. (Vgl, D. Staldtechroniken X. 429, Gesandter 1475 am kais. Hofe)

Jörg vitztum . . jagvast

in pan tun .. Ein pleul geben

Jörg von Rosemberg . . premen (S. D. Städtechr. X. 351. Auch Bunckhardt d. funfft merckisch buech $22 \mathrm{ff}$.)

\section{(Zweite Hand:)}

Jorg Haussner $V$ hallmer

Johann Weissenbach doctor
probst zn Czeiz

Jacob Roelinger $V$ Kraussbanns. (Er tritt seit 1468 in den gleichzeitigen Rathslisten auf. D. Stchron. X. 327).

$$
\text { (fol. 7 b. - Erste Hand:) }
$$

Künig zo Beheim . . Antvogel kunig Wentzlaw zu Beheim . . Adler kund oder gut freund . . Bynmeiss kadoltzburg . . Baryss kunig von franckreich . . Emerling keyserlich gebot und verbotbrief.. Greyfen korn und ander getraid . . kalb kurfursten .. korfannen kornneuburg . . kornacker (Vgl. Hassel. holdt I. 250) kuniglicher wirdi . . Coriander kuntschaft . . Badhattel kaufft . . pfannholtz keyser . . Sperber kung zu polan .. Sley kung Matheis zu Ungern . S Salm (1458-90)

kaiserlich jnsigel .. tisch

kitzing . . unru

keyserlich ladung . . Waidman

keyserlich hof .. Weysspfert

kinder . . Vogel

kam oder komen ist . . III Aicheln

keyserlich panir . . hochflüchte

klein .. VII von Schellen

(Dritte Hand:)

kriegsleufft $V$ gevaterschafft

$$
\text { (fol. 8a. - Erste Hand:) }
$$

Land ob der Enns . . Au

Lehen . . Ampferwasser

Land auf der Steyrmarck . . Bald

Lantzhut . . flachsland

Lang vergeben Red und wort . . fürbank

Lantfride . . gemein

Ludwig pfintzing . . gesell (S. D. Stadtechron. $X$. 396).

Langsam oder nit furderlich .. Grauf Landkomenthur zu Ellingen . . Garten. (Dass ein Landkomenthur zu Ellingen ein vertrauter Rath des Kurfüsten Albrecht Achills war, ergibt sich aus Burkhardt d. funfft merckisch Buech 8.7 ff.).

Ledig gesagt . . geweyst

Laugingen . . hohenstat (S. Hasselholdt II. 588 ff.)

Letzet . . hincket

Landgrauf zu Hessen . . kugelhsupt

Land und leute . . lautten 
Legat ron ferrer . . lottervall. (Als Jegat wird ein Bischof von Ferrara erwähnt. D. Städtechron. XI. 757. Ebenso V. 317 beim J. 1467.)

Landschaft z.u Österreich . . Coröl

lehenschaft . . pfaffen

Ludwig von Eyb .. leythund (der bekannte Staatsmann unter Albrecht Achill und seinen Söhnen).

lauff . . umbkraiss

Lintz . . Weysshofen

leib und gut nach allem vermogen $\}.$. Zosetzen

loch . . Kroppel

\section{(Zweite Hand:)}

lutz Schot $V$ hecktor (als Kriegshauptmann der Nürnberger erwähnt 1472. S. D. Städtechr. XI. 469. Frăher er wähnt [1462] bei Stalin, W. Gesch. III. S. $521 \mathrm{ff}$.)

ladung $V$ latern

$\left.\begin{array}{l}\text { laur dem man die finger } \\ \text { abgehauen hat }\end{array}\right\}$ losshaubt lauffertor $V$ glassberg

$$
\text { (fol. } 8 \text { b. - Erste Hand:) }
$$

Meister Ulrich Rieder . . Coppaun. (Am kaiserlichen Hofe. S. D. Stchron. II. 230; X. 407. - HasselholdtStockheim I. 133; II. 291. Dom probst. Bei Kluckhohn Ludw. d. R. S. 185 wird er einflussreicher Rath des Kaisers genannt.)

$\left.\begin{array}{l}\text { Matheis Sluder der hertzogen } \\ \text { von Müncben rete }\end{array}\right\}$. hilff Mit recht oder einicherlai $\}$. . krautt
ander sachen

Menigklich von iren wegen .. linsen

Maister hans von horbe . . lorper

Maister heinrich Imhof . . Maulwerff

(D. Stchron. X. 36, 37)

Michel paumgartner . . Maulpandt

Merhern . . Miltan

Mathes keyser . . Mucken

Maister hans Robein .. Marchwein

Marggrave zu Baden .. Norhan
Maister Sigmund drechsel . Nachtigall. (Am kais. Hofe. Gewöhnlich Dresch. ler geschrieben. S. D. Städtechron. 404. Vgl. auch Hasselholdt - Stockh. II. 699.)

Maister harttung .. Olper. (Damit wird der bei Müller Reichstagsth. IV. 141 als kais. Rath genante M. Albrecht Harttung gemeint sein.)

Marggrave Albrecht . . pfau (Albrecht Achilles von Brandenburg)

Maister Mertein Mayr . . polch (vielfach Rathgeber Nornbergs; in bayerischen Diensten z. B. D. Stadtechr. X. 338 u. B. w. Vgl. HasselholdtStockheim II. 317 ff.)

Mulhausen die statt . . peltzer

Marggrave fridrich .. Rottelwey (Kurfürst Friedrich II. von Brandenburg)

Marggrave Hans . Renck. (Wahrscheinlich Johann der Alchemyst, Bruder Friedrichs II. und Albrecht Achills [1440-67]).

Manicherlai were . . Schimpf

Maiestat insigel . . Schachzagel

Maister hans pistoris . . Zigerner

Metz . . kartenspil

Marter . . hitze

Muntz .. Spiegel

Mit allen sachen . . VI hertzen

$$
\text { (fol. 9a:) }
$$

Nachkomen am Reich .. Arabisch gold Nachparschaft . A Aubenteur

Neuenstatt jn Österreich . . Gewitz

Niclaus Gross .. Grünling (s. D. Stchron. X. 308 u.ff.)

Niderleg . . Gerstenwasser

Notari . . Gertner

Niclaus Muffel alt . . Golias. (Wurde nach dem bekannten Processe 1469 hingerichtet. S. Deutsche Studtechr. XI. 754 ff.)

Neuspital . Gerstenacker

Nach gestalt der sachen für $\ldots$.. hemelgut vernomen ist

Nacht . . hardörpfel 
Nit clein sunder gross vertraun auch unvergessen aufsehen .. leb zu haben

Name . Mitfüchsel

Neuenstatt an der Aysch . Nachtrab

Niderligen oder vahen . . Padstube

Noten oder trucken . . plumen zaigen

Neu bischof zu Mentz . . Rephun

$\left.\begin{array}{l}\text { Nit verwarlost sunder gut } \\ \text { erlangt sachen }\end{array}\right\}$. Saiffen

Noch . . Schmarack

Natürlicher herr . . Verweser

Nördlingen . . Widhopf

Nachtseld oder benachten . . zwagern

Nürmberg die statt . . Sampnung

Nicht oder keinerlei . . Ametist

Notturft . . X hertzen

\section{(Zweite Hand:)}

nebelwolken $V$ nynant mage zu jm kumen

$$
\text { (fol. 9b. - Erste Hand:) }
$$

Oberkeit . . fluch

Offenlich sendbrief . . gehurter hirss

Österreych Beyern Rein |. Ru

Swaben Francken Saclisen $\}$ wanye

Offenung . . Sper

Offenlich unverporgen .. Schaff

(Zweite Hand:)

Osterichs Cancelley $V$ oberlands cancelley

\section{(fol. 10 a. - Erste Hand:)}

Probst von pressburg . . Egel

pfalzgraf .. falk

poren oder erzeigen . . fürstin

pfaff Scheuch . . Gutzgauch. (Sollte da-

mit der Chorherr zu Ansbach Stephan

Scheuch gemeint sein? Vgl. Hassel-

holdt-Stockheim II. 388 n. a. a. O.)

pryssenstatt . . Ofenhausen

pollicey oder regirung . . polwerck

petter Gampp . . pfirsing grün (in d.

kais. Kanzlei 1461 beschäftigt. S.

D. Städtechr. X. 407)

porsspein . . posspauer paulus hegner .. Swertfisch

penn . . Stro

purgkhut .. Steinewerck

pirckenvelt . . Tüchlein

person . . Gadem

pfarrer zu kempten . . pfirsich kern

paumkirchner . Badkübel (Hassel

holdt 1. 254)

petter harstorffer .. Rulandt (D. Städte

chron. X. 303)

pfantschaft .. Salbe wasser

pfarrer Sebaldi .. Wildsaue

\section{(Zweite Hand:)}

patriarch ron Constantinopel $\vee$ patron pyms $\vee$ Schuld. (Offenbar eine Lin. stellung; denn unten steht schuld pyms).

$$
\text { (fol. 10b. - Erste Hand:) }
$$

Quittantzen .. Aurhan

$$
\text { (fol. 11a:) }
$$

Regenspurg . . Bücking

Reinisch gold . . fennd

Rottemburg an der thauber. . Gieyr

Reysige . . Gutzer

Reukauf und kümbernuss . . has

Renuer . . haselbaum

Rat zu Nuremberg . . krannch

Reformacion .. Luchs

Römisch Reich . . Oberman von S'schellen

Rechtlich henndel . . pretspil

Recht oder aid .. pretstein

Reinisch Stett . . Rapp

Rechtvertigung . . Rechbock

Römisch Canzlei . . Rotterkern

Reichen dann si treiben handel . . Rott zu dem, das ir scheurn steen $/$ stiglitz

Ritterschaft der neuen vereinung $\mid$. . vorin francken

(Hasselholdt I. 192, 193)

Ratsfreunde . . Willing

Rotweyl die Statt . . pin

Rathaus... Weintruben

Reichstett in Elsass . . küng von hertzen

Richtigung .. Dachs

Reych Stett . . küng von klee 
Rott die Statt . . Reinpolt

Romreyttigk . . Reiben

Rechtpot oder ausstrag.. VIII Aicheln

\section{(Dritte Hand:)}

Ritter | überschein

Ratslag | husfaugt

(Zweite Hand:)

Rupprecht haller $\bigvee$ Eberstock. (Sehr oft erwähnt z. B. D. Städtechr. X. 303 u. s. w.; Hasselholdt I. 94 ff.)

Rechtssatz $\vee$ Brücken

\section{(fol. 11 b. - Erste Hand:)}

Sebalt pfintzing alt .. Amster (erwähnt 1445 D. Stchr. X. 165 . 1450 ib. II. 484)

Secretirt. . Sessel

Streit . . freuden

Sorgfeltikait des rechten . . Gemss

Ser gross not tut, alle vergangen handlung und was itzunt fürgenommen, .hauetauf das höchst ganz ver swein swigen bleibe

Ser erschrocken ... jltis

Ser sorglich stende, ge- $\ldots$. keinelstanden und noch steet tier

Secret jnsigel . . Mardistelwasser

Secklein . . Mistlein

Stee oder hange . . piper

Schulde .. pyms

Stattsteur . . Raub

$\left.\begin{array}{l}\text { So lang dise krieg vorhanden } \\ \text { sind }\end{array}\right\} \begin{aligned} & \text { Rech- } \\ & \text { garn }\end{aligned}$

Salpeter . . Ratz

Swerlich allenthalben verläumet. .

Rotterstorch

Straspurg . . Schnepf

Swabach . . Stor

Sweytzer . . Stockfisch

Schaub .. Sun

Sachen und geschefft . . Salbey

Sweinfurt . . Stul

Soldner oder Diener . . Scherstatt

+ Slewitzer. . Tritt.(Ein Kachenmeister des Kaisers Slewitzer wird 1489 erwăhnt. D. Stchr. XI. 723).
Ser bekiumert . . W'elschnuss

Stette . . Vergatterung

Stattschreiljer zu Weissemburg

Wachtel

Sorgfeltikait . . zimin

Stürmen . . zu stellen

Sloss . . Zymmerberg

Schreyber . . X Aicheln

Ser gut benügen . . IX clee

(fol. $12 \mathrm{~b}$ :)

Sachen .. III clee

Scheinbrief, daran nichts ligt . . Aff

Schedlichen. Eyl. Eysvogel

Stettlich arbait zu gepietten oder durch verwillingen ... helf-

kranch allenthalben zuze- fant greiffen

Schauffhausen die Stat, die ain bruck über . . Obennann Rein hat von hertzen

Starck und kreffitig .. Oberman von clee Spitz das Sloss . . Stumpf. (Spitz und Wachau gehorten zu bayerischen Besitzungen in Oesterreich (Nünb. A.) Steyr . . Speck (Vgl. Hasselholdt I. 157) Schuttensam .. Wurm (erwahnt als Feind der Nümberger 1461-74. D. Stchron. X. 341 ff.)

Stett $2 \mathbf{u}$ halten . . : :

\section{(Zweite Hand:)}

Spital $V$ frondinst

Steyrisch $\vee$ Blokeren cancelley

$$
\text { (fol. 12b. - Erste Hand:) }
$$

Tunowerde .. All

Totten . . kalhart

Tunau das wasser. . Badofen

Tode .. Schrannen

Türcken . . Tracken

Truhen - . verspruch

Tage oder nacht . . V Aicheln

Tausent . . Leber

Teufel . . Mangolt

Teufelmalen . . Tausentmeul

$$
\text { (fol. 13a:) }
$$

Ungchuld . . Agrest 
Vor .. Berel

Ulm . . Colmeys

Urkund făr rechtfertigung . . Erlein

Verlesen, vernomen $u$. verstanden . . Esel

Vechde und krieg . . freuntschaft

Virgili der schreyber . . fink

Verharren oder verzug . G Grefin

Vasnacht . . hermlein

Vertrag .. hessen

volzogen und vollendet wurt . . kitz

Ungunst oder unwillen . . loseysen

Vollmechtigen anwalt . . petterlein

Versigelt brief u. urkund . . plaupfert

Ursach . . penet

Verschreibung .. patene

Unversert geantwort . Rose

Ungutlich geschehen .. Rotterbeher

Vidimus . . Salz

Urtailer . . Stöck

Vorstmaister ampt . . selige statt

Ulrich wetzel .. Taube (sicher identisch mit d. kais. Kanzler Ulrich Weltzli. Vgl. D. Stchron. X. 398,407 . S. uber ihn Stälin Wirt. Gesch. III. 455).

nō Untreu oder verleytung .. Weichsel

Ulrich Artzt . . finckenau (s. über ihn D. Stadtechr. X.197. A. 2).

Vom leben zum tod .. Volbad

Usmer .. Unmuss

Unrecht . . IIII hertzen

nū Untreu .. IIII Schellen

Unrat .. UI Schellen

Verhindert oder geirret.. II Schellen

Uberkomen, vertragen oder
vereinigt. . X clee

Vorcht oder sorg . . VIII clee

Vermeiden oder abwesen . . VI clee

Vertigung . . IIII clee

Verdriess .. II clee

Ungezweifelt . . : :

Vermerkt, erlemnt od.verstanden $\bullet$

Veind .. Ber

$$
\text { (fol. } 13 \mathrm{~b} \text { :) }
$$

Verporgenlichen . . Cromat

Verlangen .. Diemant
Unbillichs oder unfreuntlichs . . holz. hauen

Vencknus .. Vogelhaus

Unbillichkait . . Saffran

Verdechtlich .. Virdung

Ungelt . . Ungeschmach

Unwiderruflichen . . VIII schellen

(Zweite Hand:)

Wagen $V$ gezenge

Vollung $V$ traben

Urtel $V$ Eye

\section{(Erste Hand:)}

Wilhelm von buchen .. Aich

Wilhelm Stor . . Grasmuck

Wochen .. holzapfel

Welde . . hoeneich

Wo man nit verswigen wer das solchs ser schedlich . . lint scheden bringen wurde wurm

Wendelstein . . Mistel

Weysung . . Nuss

wolfail oder ein pfenwert . . pfann

Wienn . . rotttroschel

weren verprannt, verheret |. Rotter oder verderbet 1 kimerling

Wo es nit anders wolt sein, ee wolten sie den Rat zu- . . Rotag saupt hertzog ludwigen
zu veind haben

$$
\text { (fol. 14a:) }
$$

Wo wege mit fugen mochten funden werden, das will- . . Rottfarung geschehe und durch begsteltr. die vinger gesehen

Windsheim .. Sittich

Weyssenburg .. Storch

Wilhelm loffelholtz .. Schraub (oft er wahnt z. B. D. Stchron. X. 416 u. s. w.) Wunderlich oder gremsig . . Schüssel Wagenburgk .. virrade

Wort .. Weyssruben

Wildpann . . Umblauff

Widerwertikait . . Wirm

Weissemburg am Rein .. Weyrauch

Warnung . . Zuckerwasser 
Weysen oder unterrichtung . . III Aicheln

Wilhelm Derrer . . freynut (mehrfach erwahnt in d. Nürnb. Chroniken z. B. D. Stchron 434 u. s. w.)

Wein .. hasengarn

Wege oder Strass . . kalkus

Wachen .. laub

Walld das Sloss .. Nagel

Wienig odes lutzel . . Osterwein

(Dritte Hand:)

Weihannt koneck $V$ Elias. (Vgl. oben unter Elias. Die folgende Zeile gehört dazu. Rheinscbwalbe $=$ Bischof von Metz.)

reinswalben Canceller

(Zweite Hand:)

wucher $V$ mist

her Wilhelm von Auersburg $V$ holper

(fol. 14 b. - Erste Hand:)

Zebenden dn mitssmpt dem j.. Brauguldin opfer pfenning nellen

Zweyen Ratten . . kolben.
Zaiger oder antworter . . lepart

Zway swert . . Malmaseyer

Zornig ist . . Mader

Zugericht oder gevertigt . Ruckinbrot

7u sorgen, das des Rats armleut, auch der kauff- . Rotter man beschedigt werden rab möcht

Zu tun nymant gestatten . . Raben

Zölle . . Stein

Zu schonen . . Schar

Zwifach . . tischtuch

Zum Gostenhof . . Wisbaden

Zittedell Jud .. Unglaub

Zu Uberziehen .. Z Zu Eren

Zu erlangen oder zu er- I. . IV Aiche raichen

len

Zugeben oder zugesagt . . II hertzen

Zu rotten . . Beinschruben

Zu scheubt jn sein hilf wider $\}$. . Griel

$\mathrm{Zu}$ diser zeyt oder stund . . Regina

Zollstett . . Steingrub

Zu fügen oder zu schicken .. Scher

Einige Bemerkungen drängen sich unwillkürlich auf. Sowohl die eigentlichen Ausdrücke auf der linken Seite sind im Grossen und Ganzen alphabetisch geordnet, wenn auch die Reihenfolge nicht gerade peinlich bestimmt ist, wie auch die geheimen Zeichen auf der rechten Seite innerhalb eines jeden Buchstabens. Denn das geht doch über den Zufall hinaus, wenn beim Buchstaben $\boldsymbol{A}$ die Worte Abguck, Affeltar, Coppaun Wasser, Gans, Glas, Jeger u. s. w., beim Buchstaben B Behmisch, Beystadel, Citron, Esch, Erbotz, Eneas, Fürst u. s. w., beim Buchstaben $\mathrm{C}$ die Worte Arbais, Berlinkrantz, Comat, Kiefarbais u. s. w., beim Buchstaben D Ainküre, A, B, Darmvol, Geyss, Hering, Herz u. s. w. auf einander folgen. Die Aufzählung liesse sich mit dem gleichen Ergebniss bis zum Buchstaben Z durchführen; aber die angeführten Beispiele erhärten die Behauptung wohl schon zur Genüge. Dabei springt aber ebenso deutlich in die Augen, dass die Zusätze zweiter, dritter und vierter Hand dieser Regel nicht unterworfen sind; da geht es, um irgend einen Buchstaben herauszugreifen, bei $H$ bunt durcheinander : phasan, 
'Tubias, hoenschein, frischier mut, hopfgartner, platzmeister.' Aber allerdings auch die erste Hand hat sich genöthigt gesehen, das Alphabet nach Erschöpfung des Wortvorraths wieder von neuen zu beginnen. Der Buchstabe $G$ wird das klar machen. Er beginnt mit Amsel, Antlatz, Alber, Biber und geht fort bis zu Zamerbere. Ziella, Zwirn; darauf folgen wieder von nenem 9 Aicheln, 7 Clee. $\cdot \cdot$, erper, vynstern (finstern), Otter, Roth, Wildper, Weissrosen. Ich wage nicht, die Frage 7. u untersuchen, ob dieser Umstand darauf hindeutet, dass die Notizen der ersten Hand ursprïnglich auch wieder in zwei Gruppen, in eine ältere und eine jüngere, die vielleicht eben aus verschiedenen Jahren herstammen, zu scheiden seien. Denn allerdings haben wir es ja bei der Arbeit der ersten Hand nicht mit einer Urschrift, sondern, wie oben schon bemerkt, mit einer Abschrift zu thun, die natürlich die Kennzeichen der verschiedenen Einträge verwischt hat. Es würde das eine ebenso schwierige und vielleicht ebenso wenig lohnende Untersuchung sein, wie wenn man feststellen wollte, ob die im Jahre 1500 gebrauchten Ausdrücke wie Sperber, Falke, Pfau u. s. w. eine bestimmte Beziehung zu der damit bezeichneten Persönlichkeit1) oder zu ihrer amtlichen Stellung (grosse und kleine Vögel? 2) haben, und ob auch bei diesem Verzeichniss für den Kardinal von Augsburg absichtlich die Bezeichnung Rothamsel, für Herzog Albrecht IV. von München Frischer Muth, für Lutz Schott den Hauptmann Hektor, für Doktor Kilian (von Bibra) Ratgebe, für Ludwig von Fyb Leithund gewählt ist.

Dagegen ist unbedingt nicht $z u$ umgehen eine Untersuchung darüber, welcher Zeit dieser Schlüssel angehört. Denn dass er auf diejenigen Aktenstücke, bei denen er jetzt liegt, nicht passt, hat sich theils oben schon ergeben, theils lehrt es der erste Blick auf die Personen, die darin vorkommen. Und eben diese Personen müssen auch den ersten Anhalt bieten, um eine Zeitbestimmung zu gewinnen. Der terminus a quo würde zunächst annähernd festzustellen sein aus der Erwähnung des kung Mattheis zu Ungern. Da diese Notiz der ersten Hand und zwar unzweifelhaft dem Grundstocke der Bezeichnungen angehört, so ist wohl nicht daran zu

') Auerhahn-Albrecht der Beherzte? Pfau die stolzen eitlen Markgrafen von Brandenburg?

2) Sperber, Falke, Pfau, Auerhahn etc. bezeichnen die Fürsten, Zeisig, Koblmeise geringere Persönlichkeiten. 
zweifeln, dass dieser Schlüssel vor den Jahre 1458, dem Jahre des Regierungsantritts des Königs Mathias, nicht zusammengestellt sein kann. Dass wenige Personen darin vorkommen, die zu dieser Zeit längst aus dem Ieben geschieden waren, wie z. B. König Wenzel von Böhmen, kann uns in dieser Ueberzengung nicht beirren, da ja nach ibrem Tode sehr wohl noch von ihnen die Rede sein konnte. Dagegen würde an diesem Zeitpunkt allerdings nicht festgebalteu werden können, wenn Persönlichkeiten in dem Verzeichniss aufträten, die entweder später geboren oder doch mit einem Titel bezeichnet würden, den sie erst später erlangten. Dann würde die Entstehungszeit noch um einige Jahre später zu datieren sein. Da nun ein Herzog Ott erwähnt wird und diese Notiz hinter Herzog Ludwig von Bayern und Herzog Ludwig von Veldenz sich kaum auf einen andern Fürsten als den Herzog Otto von Neumarkt, der seine Regierung erst 1461 antrat, beziehen kann, so wird man den terminus a quo bis zu diesem Jahre zu verschieben haben. Ein Grund, ihn noch später anzusetzen, scheint nicht vorzuliegen. Denn dass z. B. Herzog Viktorin, der Sohn Georgs Podiebrad, der erst 1462 das Herzogthum Troppau erhielt, vorkommt, würde nichts zu bedeuten haben, da dieser junge Fürst in den Urkunden des Jahres 1461 (vgl. z. B. beim 15. Nov. 1461 Hasselholdt-St. II. 502 u. a. v. St.) schon regelmässig Herzog genannt wird. Ebenso würde die Erwähnung des Herzogs Ernst von Sachsen, der seine Regierung 1464 antrat, deswegen nicht dagegen sprechen, weil, wie oben bemerkt, von der ersten Hand Herzog Friedrich von Sachsen geschrieben war, und die Verbesserung von einer anderen Hand herstammt. Etwas schwerer könnte ins Gewicht fallen, dass der Name Schuttensam erwähnt ist, von dem ich eine gewisse Bedeutung für Nürnberg erst seit dem Jahre 1464 nachweisen kann. Aber entscheidend wird bei der Möglichkeit, dass die Streitigkeiten schon früher begonnen haben, dieser Umstand um so weniger sein, als so mancher andere Grund noch für das Jahr 1461 als Anfaugstermin spricht. 1)

Wenn es eigentlich auch selbstverständlich ist, dass diese Geheimschrift nur angewendet worden sein wird, wenn es sich um besonders wichtige und geheime Dinge handelte, so verdient es

1) Die Erwähnung Herzog Albrechts von München, der in die Mitregierung 1465 (vgl. Hasselholdt-St. I. 333) eintrat und des Grafen Ott von Henneberg, der in demselben Jahre zu regieren begann, streitet nicht dagegen, da beide erst von späterer Hand eingetragen sind. 
doch noch besonders herrorgehoben zu werden, dass folgende Ausdrücke in dem Schlüssel rorkommen: ser gross not tut, alle rergangen handlung und was itzunt furgenomen, auf das höchst ganz verswigen bleibe, und ferner: wo man nit verswigen wer, das solchs ser schedlich scheden bringen werde. Nun hatten die Nürnberger aber gerade in den Jahren 1461/62 einen solchen geheimen Handel vor, von dem vor allen ihr Nachbar Albrecht Achilles nichts erfahren durfte. Als nämlich der Reichskrieg von Kaiser Friedrich IU. gegen Herzog Ludwig den Reichen von Bayern erk]ärt worden war. suchte der hohenzollernsche Markgraf, der neben dem Grafen Ulrich ron Württemberg und Karl von Baden zum Reichsfeldherrn ernaunt wurde, ganz besonders den Beistand der Reichsstädte zu gewinnen. und er brachte vom Kaiser die schärfsten Mandate gegen sie aus. Für Nürnberg war die Verlegenheit gross; um keinen Preis wollte man es mit dem bisherigen Bundesgenossen, dem Herzog Ludwig. verderben; aber andererseits war es auch gefährlich, den kaiserlichen Befehlen zu trotzen, hinter denen ja der thatkräftige Markgraf Albrecht stand. So entschloss sich denn der Rath, eine Gesandtschaft zum Kaiser abzuordnen, um auf irgend eine Weise Befreiung von der Verpflichtung, an dem Reichskriege theilzunehmen. zu erlangen. Bei Friedrich III. war mit namhaften Summen schon etwas auszurichten und wirklich hatte die Gesandtschaft Erfolg: sie brachte ein vom 7. Sept. 1461 datiertes Schreiben des Kaisers aus. das die Nürnberger von jeder Kriegsverpflichtung entband. Aber nicht zufrieden damit erwirkten die Nïrnberger durch eine zweite Gesandtschaft noch eine goldene Bulle, die sie auf 18 Jahre von allen kriegerischen Leistungen für das Reich befreite. Dieses Dokument ist zwar aus dem Jahre 1452 datiert; aber der verstorbene Professor von Kern hat im 10. Bande der deutschen Städtechroniken (S. $408 \mathrm{ff}$.) mit unwiderleglichen Gründen bewiesen, dass der Kaiser und seine Kanzlei sich einer Fälschung schuldig gemacht und die Orkunde, die im Winter 1461/62 ausgestellt wurde, auf den 23. Juni 1452 zurückdatiert haben. Gar so auffallig ist dieses Verfabren bei Friedrich 1lI. nicht; er hat auch sonst derartige Manipulationen und zwar immer mit der nöthigeni Vorsicht (z. B. musste genau der Aufenthaltsort für den falschen Termin, den man wählen wollte. von der Kanzlei festgestellt werden) vorgenommen. 1) Aber aller-

1) Vgl. das oben erwähnte Programm: Aufnahme der fränkischen Hohenzollem in den schwäbischen Bund. Berlin 1880. S. 19. Anm. 4. 
dings lag ihm dann immer riel daran, dass die Sache geheim gehalten wurde und über den Kreis der Betheiligten nicht hinauskam. In diesem Falle wird er ganz besondere Heimlichkeit zur ersten Bedingung gemacht haben. Markgraf Albrecht durfte um keinen Preis etwas davon erfahren, wie ihn der Kaiser hinterging, da es in eigensten Interesse Friedrich III. gelegen war, dass er den gegen Ludwig von Bayern beschlossenen Krieg mit aller Kraft führe. Daher gab sich der Monarch, selbst als er den Nürnbergern schon die Exemption von dem Reichskriege zugestanden hatte, noch den Anschein, als komme er den dringenden Bitten des Markgrafen nach und leite den Prozess gegen Nürnberg wegen Ungehorsams vor dem kaiserlichen Kammergerichte ein. Daraus wird sich für die Nürnbergischen Gesandten auch die Nothwendigkeit ergeben haben, die Berichte, welche sie in dieser Angelegenheit an den Rath richteten, in einer Geheimsprache abzufassen, für den Fall, dass einer ihrer Briefe in die Hände des Markgrafen, den sie durch Betheiligung an den zur Beschliessung der Kriegshilfe ausgeschriebenen Städtetagen in Sicherheit zu wiegen suchten, auf irgend eine Weise geriethe. - Versuchen wir nun den Beweis anzutreten, dass ein grosser Theil der in dem vorliegenden Schlüssel aufgeführten Ausdrücke gerade auf diese Verbältnisse passt, so dass er dem an den Kaiser abgeordneten Gesandten Nürnbergs, Jobst Tetzel, als „vocabulari“" mitgegeben worden sein könnte. Den ersten Anhalt werden uns die Personen darbieten. Tetzel, der in dem Schlüssel cromatfogel genannt wird (vielleicht weil er verborgenlichen $=$ cromat mit einer vertigung abgesendet wurde), hat in einem erhaltenen Rechnungszettel (Deutsche Städtechron. X. 406) die Ausgaben (den costen oder das gelt $=$ underman von clee) vermerkt, die er im Jahre 1461 am kaiserlichen ${ }^{* H o f e}$ gemacht hat; aber sie sind auch im „Register des Ausgebens während des Krieges von 1461" aufgeführt. Die darin vorkommenden Ausdrücke liessen sich recht wohl in Geheimschrift ausdrücken. Da treten neben dem Kaiser (sperber), der 3000 Gulden (30 Karpfen) rheinisch Gold (fennd) erhält, Meister Sigmund Tröschler (nachtigall), Wolfgang Vorchtnauęr (norff), der Bischof von Gurk .(Zagelmaiss), Ulrich Weltzli (taube), Meister Ulrich Riederer (coppaun), Peter Gamp (pfirsing grün) am kaiserlichen Hofe (weisspfert) theils in der römischen (rotterkern), theils in der österreichischen Kanzlei (oberlands cancelley) auf und erhalten erung oder schenk (walfisch). Auch 
Wagner :

die guldin pull (otter) und das vidimus (salz), das Jobst Tetzel zu Grätz (Gegler) anfertigen liess, die collationirte abschrift (swartzpfert) dieser versigelt brief und urkund (plaupfert), an die der maiestat insiegel (schachzagel) oder das kaiserlich insigl (tisch) gehängt wurden, lassen sich belegen. Ebenso wird der Abt von St. Egidien. der ebenfalls ein Vidimus der goldnen Bulle ausstellte, als abguck erwähnt. Dass der alt bischove zu Mentz (Gans) und später der neu bischof zu Mentz (rephun) genannt werden, liesse sich wohl zwanglos damit erklären, dass in eben jener Zeit (Anfang Okt. 1461) Graf Adolf von Nassau an Stelle Diethers von Isenburg vom Papste zur erzbischöflichen Würde erhoben worden war. Der Kardinal vou Augsburg (rottamssel; ebenso bei bischof $\%$ u Augspurg) musste auch neben den Bischöfen von Bamberg (sprintz), Würzburg (galander) und Salzburg (rotterthauber) erwähnt werden, da sie insgesamnt zur bayerischen Partei gehören. (S. Hasselholdt-Stockheim I. 155 ff. 237.) Der Bischof von Eichstädt (henfling) darf nicht fehlen, da er, der frühere Feind Ludwigs des Reichen, später eine vermittelnde Stellung. einnahm. (S. z. B. die päpstliche Bulle an ihn bei Hasselholdt II. 640.) Der Bischof von Passau (thauber) spielt neben den Kardinal von Augsburg und Ludwig dem Reichen 1461 eine Rolle in den Streitigkeiten des Herzogs Sigmund von Oesterreich (tenlein) mit dem Bischof von Brixen. (Hasselholdt II. 385.) - Dagegen stand der Bischof you Metz (reinswalb; auch sein Kanzler Weihannt Konek = Elias ist von späterer Hand hinzugefügt) auf der Gegenpartei; er wurde ja mit dem Grafen Ulrich von Württemberg (raiger) und seinem Bruder, dem Markgrafen von Baden (norhan) in dem Gefecht von Secken. heim (30. Juni 1462) von dem Pfalzgrafen (falk) 1) gefangen genommen. Die Erzbischöfe von Köln (haselhun) und Trier (gruntel), der Bischof von Freising (citron) wurden im März 1462 vom Kaiser aufgefordert, gegen die bayerische Partei zu Felde zu ziehen (Hasselholdt I. 235) und bis zu dieser Zeit weilte Jobst Tetzel als Gesandter am kaiserlichen Hofe. Ueber die Beziehungen des Bischofs von Agram (rotkopf) zu diesen Verhältnissen giebt die Notiz Aufschluss, dass er 1461 als Gesandter Albrechts zum Herzog Ludwig ging. (Hasselh. I. 144.)

1) Merkwürdig genug ist es, dass Albrecht Achilles in seinem bei Hasselholdt I. 247 abgedruckten Schreiben an den Markgrafen Marx von Baden auch sagt: das ir euch der ding entslahet, was euch des zu swermütigkeit dinen moge und euch trostlich gegen dem falck stelt u. s. w. Da scheint mit dem Falken ja auch der Pfalggraf gemeint zu sein. 
Dass auch der Papst (esch) aufgeführt wird, ist an sich erklärlich: wenn aber gerade in dieser Zeit die Gegner des Böhmenkönigs denselben ersuchen, dass er „bei dem pann geböt, das kain man in der Cron zu Beheim, zu Merhern noch in der Slesien wider den kaiser noch sein Helffer sey" (Hasselholdt I. 151.181 ff.), so ist man allerdings versucht, diese Worte nach Anleitung des Schlïssels zu übertragen: babst esch bei dem höchsten beptlichen panne kue gebotten heu, das kain man in der cron zu Beheim bernlinkrantz, zu Merhern miltau, noch in der Slesien wider den kaiser sperber, noch sein helffer habern sei. - Von weltlichen Herren werden manche ohne nähere Bezeichnung erwähnt, wie z. B. Pfalzgraf (falk), Jarkgraf zu Baden (norhan), Landgraf zu Hessen (kugelhaupt), König von Böhmen (antrogel), Polen (sley), Frankreich (emerling); diese werden also zur Feststellung der Zeit, in welcher der vorliegende Schlüssel entstanden ist, nicht weiter herangezogen werden können. Allerdings verdient hervorgehoben zu werden, dass der Pfalzgraf, der Markgraf zu Baden, der König von Böhmen in die Politik der Jahre 1461/62 tief verflochten sind und dass nicht allzu lange vorher von einem Hereinziehen Polens, Frankreichs, Burgunds, der Schweiz und Venedigs in diese Angelegenheiten auf dem Prager Tage 1461 die Rede gewesen war. (Hasselholdt II. 493 ff.) Besonders beachtenswerth erscheint, dass in den Notizen erster Hand nur die Herren von München (begsteltz) erwähnt werden; das kann sich doch nur auf das gemeinsame Regiment der Söhne Albrechts III. des Frommen beziehen, also entweder nur auf die Jahre 1460-64 - da regierten Johann und Sigismund allein - oder höchstens 1460-68. Das erstere ist wahrscheinlicher, da eine spätere Hand erst ausdrücklich Herzog Albrecht IV. (frischermut), der 1464 schon seine Zulassung zur Mitregierung erzwang, hinzugefügt hat. Als ihr Rath wird Matheis Sluder bezeichnet; mit diesem Vornamen findet sich in dem mir zugänglichen Material kein Staatsmann; wohl aber wird ein Peter Schluder in den letzten Regierungsjahren Albrechts III. und in den ersten Albrechts IV. genannt. (Hasselholdt I. 24.) Dass sie in den Wirren der Jahre 1461/62 sehr häufig auftreten, ist aus ihrem Verhältniss zu der Hauptperson jener Streitigkeiten, dem Herzog Ludwig dem Reichen (plaufuss), sehr erklärlich. Zur bayerischen Partei gehörten ausserdem des Kaisers feindlich gesinnter Bruder 
Herzog Albrecht ron Oesterreich (pluteisicken) mit der Landschaft zu Oesterreich (coröl) und mit dem Könige Mathias von Ungarn (salm), mit Herzog Sigismund von Oesterreich (tenlein), der Gesellschaft St. Georgenschild (hasel); ausserdem stand Herzog Ludwig in Verbindung mit den Herzögen Wilhelm (turteltaub) und Friedrich von Sachsen (fasan), 1) dem Herzog von Burgund (kraue), mit dem Pfalzgrafen (falk). Der Pfalzgraf selbst stand wieder im Bündniss mit dem Landgrafen zu Hessen (kugelbaupt). (Hasselholdt J. 155.) Später (1462) tritt noch Herzog Otto von Mosbach (rottauge) hinzu (Hasselholdt I. 235), während Herzog Ludwig von Veldenz (pirickhan) der Gegenpartei angehörte. Diese setzte sich zusammen aus den Markgrafen von Brandenburg, Friedrich II. Kurfürsten (rottelwey), Albrecht Achill (pfau), Johann dem Alchemysten (renck), dem Markgrafen von Baden (norhan), den Grafen Tlrich (raiger) und Eberhard (neunaug) von Württemberg, dem Grafen Ulrich von Oettingen (amsel), Grafen Hans von Wertheim (biber) u. a. Dass auch Herzog Virtorin, der Sohn Georg Podiebrads, der von 1462-1500. Herzog von Troppau war, (unter dem Namen ispen) auftitt, spricht auch für die Zeit 1461/62; denn im November 1461 sollte Viktorin - der in den Urkunden, wie oben bemerkt, damals schon Herzog genannt wurde - den Krieg gegen Brandenburg eröffnen. (Hasselh. I. 206.) Bei den minder bedeutenden Persönlichkeiten welttichen Standes ist es nicht in allen Fällen möglich, die Beziehungen anzugeben, welche ihre Aufnahme in dieses Verzeichniss bewirkt haben mögen; es muss genügen, -wenn wir nachzuweisen vermögen, dass ihr Vorkommen nicht geradezu der aufgestellten Vermuthung widerspricht. Von den Grafen sind schon einige erwähnt worden; es bleiben noch übrig: Graf Rudolf von Sulz = alber (das könnte sehr wohl der kaiserliche Rath sein, an den sich 1465 Albrecht IV. von Bayern-München mit der -Bitte wendete, beim Kaiser einen Befehl zu erwirken, dass seine Verwandten, Pfalzgraf Friedrich, Herzog Ludwig und die gemeine Landschaft eine Versöhnung zwischen ihm und seinen Brüdern einleiteten); Graf Haug von Werdenberg = Günther von Werd (unzweifelhaft jener einflussreiche Staatsmann, der unter Friedrich III. und Maximilian I. eine sehr bedeutende Thätigkeit entwickelt hat; gerade in den Angelegenheiten der Reichsstädte ist or häufig verwendet worden); ${ }^{2}$ ) -

1) Die sächsischen Herzöge traten dann allerdings zur Gegenpartei über.

3) Vgl. auch mein Programm : Die Aufnahme der frainkischen Hohenzollern in den schwäbischen Bund. Berlin 1880. S. 16. 
Graf Michael ron Maidburg = grasmair (ich beziehe diese Notiz auf den im Gefolge des Kaisers 1444 erwähnten Hofrichter, der eigentlich Burggraf von Magdeburg und Graf zu Hardeck war, und der schon in dem genannten Jahre neben anderen kaiserlichen Hofbeanten von der Stadt Nürnberg ein Geschenk [,ein vergulten verdeckten ausgestochen pecher"] empfing); - Graf Hans ron Eberstein $=$ gauffer (über ihn rermag ich nichts beizubringen); ${ }^{1)}$ - Graf Schaffrid von Leiningen $=$ wespen (darunter wird ein kaiserlicher Rath dieses Namens zu verstehen sein, der 1463 sich mit dem Pfalzgrafen einigte und den die Stadt Nürnberg laut Rechnungsbuches im J. 1471 neben anderen Räthen Friedrichs III. mit 20 Gulden beschenkte). - Daneben kommen noch ror von niederem Adel : Herr Hanns von Frauenberg zu Brunn = hausknecht (er war 1461 unter den Räthen, welche die Brüder Johann und Sigismund von Bayern - München nach Laugingen sendeten, um eine Binigung mit ihren Verwandten, dem Herzog Otto von Mosbach und Herzog Ludwig dem Reichen, wegen der Salzstrasse herbeizuführen, und 1462 wurde er [Hofmeister war er damals] an den kaiserlichen Hof abgeordnet); - Herr Bernhard von Schönberg = hecht (über diesen vermag ich nichts Sicheres anfzufinden; neben den fahrenden Mannen des oben erwähnten Grafen von Maidburg wurde allerdings 1442 von der Stadt Nürnberg auch der Lautenschläger eines Herrn von Schönberg belohnt; da aber der Vorname fehlt, bleibt die Beziehung unsicher) $;_{1}{ }^{2}$ ) - Herr Georg von Giech, Domherr zu Würzburg = maneser (unzweifelhaft derselbe, der 1460 an der Richtung zu Roth, allerdings als Abgesandter des Bischofs von Bamberg, betheiligt war); - Herr Heinrich Marschall = regelbirner (man kann kaum im Zweifel sein, dass man diese Bezeichnung auf den 1461 oft erwähnten Heinrich von Pappenheim [vgl. z. B. D. Stchron. X. 397]. Reichserbmarschall, und nicht auf Heynrich marschalck der jung, her Hansen sun, der unter den in der Schlacht bei Gingen 1462 Gefallenen erwähnt wird, beziehen soll); ${ }^{3}$ ) -- Herr Michel von

1) Ein Loren\% ron Eberstein wird unter den Feldhauptleuten Albrecht Ichills erwähnt. \&. Hasselholdt I. 245.

$\left.{ }^{2}\right)$ Ohne grosses Gewicht darauf legen zu wollen, möchte ich doch nicht unerwäbnt lassen, dass die Bezeichnung becht daraus entstanden sein konnte, dass unter den damaligen kaiserlichen Protonotarien sich einer Namens Hecht befand. Vgl. D. Stchron. 3, 399.

3) Ob Heinz Seybot hier oder erst später zu erwähnen sei, vermag 
Saunsheim = hausvol (über dieses Mitglied eines treu zu den brandenburgischen Markgrafen stehenden Geschlechtes vermag ich keine genaue Auskunft zu geben); ${ }^{1}$ ) - Herr Georg von Stein $=$ tann (offenbar der Kanzler des oben erwähnten Erzherzogs Albrecht ron Oesterreich, der 1461 mehrfach, z. B. als Gesandter an den Hof des Königs Mathias von Ungarn [Hasselholdt I. 202] erwähnt wird); - Herr Ulrich von Grafeneck = abraumer (er wird als kaiserlicher Rath im J. 1462 bei dem Wiener Aufstande erwähnt und ist später [1467] kaiserlicher Bevollmächtigter auf dem Reichstage zu Nürnberg); - Herr Ludwig von Eyb = leithund (dieser bekannte brandenburgische Staatsmann, der von 1440-1502 thätig gewesen ist, gehört unzweifelhaft hierher, da er die Ritterwürde mit dem Prädikat „Herr" besass); - Jörg von Wendingen = wiltfang (er gehörte im Juni 1461 zu den Räthen, welche Markgraf Albrecht zu Herzog Ludwig von Bayern sendete, um einen Ausgleich zu versuchen); - Jörg von Rosenberg = premen (er mag auf brandenburgischer Seite gestanden haben; 1478 bestand er einen Zweikampf zu Ansbach mit Simon von Stetten); - Herr Jakob Rauscher, Pfarrer zu Weissenburg = Jacob Samuel (über ihn habe ich ebensowenig etwas gefunden wie über) Herr Christoph Morsberger = spieling und Herr Wilhelm Trauner $=$ weisstrapp.

Wenden wir uns zu den Gelehrten und zugleich zu den Staatsmännern meist bürgerlicher Herkunft, so begegnen uns: Dr. Hans Birkheimer = Josepp (er tritt schon 1458 als Nürnbergischer Rath am kaiserlichen Hofe auf [Hasselholdt I. 36], wird aber auch 1459 und in den folgenden Jahren als Rathsfreund erwähnt); - Dr. Kilian $=$ ratgebe (damit ist unzweifelhaft der in die Händel jener Zeit vielfach verflochtene Domherr zu Würzburg gemeint, der z. B. 1460 als Abgesandter des Bischofs von Bamberg zwischen Albrecht Achill und Ludwig von Bayern zu vermitteln suchte [Hasselholdt I. 91. Hofler, kais. Buch S. 83]); - Dr. Knorr = trapp (ebenfalls ein vielgenannter Gesandter, der z. B. $1461 \mathrm{im}$ kaiserlichen Auftrage von Albrecht Achill zu Ludwig XI. von Frankreich und dem Herzog Philipp von Burgund geschickt wurde, um der Gegenpartei jede Hilfe

ich nicht festzustellen, da er (bei Hasselholdt-St. II. 218) ein gehalbirt edling genannt wird.

1) Sollte "Michel, Herre zu Swartzenburg von Senssheim" (bei Hasselholdt I. 458) gemeint sein? Die Herren von Saunsheim = Seinsheim und die Schwarzen. berge waren nahe verwandt. Vgl. auch D. Stchron. II, 146. 
ron dorther abzuschneiden [Hasselholdt I. 202]) - Dr. Jopp = swalb (er war 1461 als Gesandter Albrecht Achills neben Dr. Peter Knorr in den Streitigkeiten zwischen Erzbischof Diether von Mainz und Papst Pius II. sowie bei den Verhandlungen mit den Reichsstädten auf einem Tage zu Esslingen thätig); - Dr. Jörg Heimburg = lıausen (jedenfalls der bekannte Gregor ron Heimburg; wenigstens bezieht Kern einen in der Deichslerschen Clironik erwälınten „doctor Jörgen", der 1461 zugleich mit dem Erzherzog Sigismund von Tyrol in den Bann gethan wurde, auch auf Gregor Heimburg [Deutsche Stchron. X. 263 Anm. 4; vgl. auch Höfler, kais. Buch S. 85]; er trat z. B. für Erzbischof Diether als Anwalt auf demselben Tage, den Dr. Knorr und Dr. Jopp besuchten, auf); - maister Mertein Mayr = polch (der vielgenannte, stets mit Reformplänen beschäftigte, auch 1461/62 oft als bayerischer Abgesandter hervortretende Staatsmann); - Heinrich Span $=$ muffeltier (dieser kaiserliche Abgesandte wird 1461 in des kaysers spruch als von den Leuten Herzog Ludwigs niedergeworfen und schwer verwundet erwähnt) $;-$ maister Hartung $=$ olper (damit ist jedenfalls der kaiserliche Rath Magister Albrecht Hartung gemeint, den Friedrich III. 1462 ins Reich um Hilfe sendete, als er von den Wienern bedroht wurde). - Von Ulrich Weltzel, Ulrich Rieder, Sigmund Drechsel und Wolfgang Forchtenauer ist schon oben die Rede gewesen, ebenso wie von Mathes Sluder. Hans Harscher wirtembergisch $=$ dorn war 1468 Mitglied der württembergischen Regentschaft; - Erasem Thorer = slech ist der bayerische Kammermeister, der 1461 (im Dezember) an dem oben erwähnten Tage zu Laugingen Theil nahm; - pfaff Scheuch = gutzgauch kommt vielfach als vertrauter Rath des Markgrafen von Brandenburg ror, z. B. am 18. Juli $1441 \mathrm{ff}$; - - Heinz Seybot = schnaugk tritt vielfach als Rath nnd Gesandter Albrecht Achills auf, z. B. 1463 bei dem Tage von Neustadt. Von einigen kann ich keine ganz bestimmten Angaben machen; einen Dr. Hayden vermag ich erst 1486 als brandenburgischen Rath nachzuweisen; 1) ein Dr. Rott wird 1468 in dem Iuffelschen Prozesse erwähnt. Ueber Meister Hans Robein, Meister Hans Pistoris, Meister Hans von Horbe, Maister Heinrich Imhof 2) habe ich nichts auffinden können.

1) Ein Eintrag in den Hersch. Büchern des Nümb. A. lantet: Mertin Heyden doctor hat den rat gesworn. Actum Onolzbach montags Galli ao. 86.

$\left.{ }^{2}\right)$ Ein Heinrich Imhof wird 1439 als Freischöffe erwähnt. D. Stchron. X. 36. 37. 1443 kommt er bei dem Zuge nach Lichtenburg vor. 1449 ist sein 
Selbstredend treten viele Nürnberger in dem Verzeichnisse auf. Ruprecht Haller = eberstock trat 1460 neben Wilhelm Löffelholz $=$ schraub als Gesandter Nürnbergs behufs Vermittlung zwischen Albrecht Achill und Ludwig von Bayern auf. Er war bei der Rother Richtung ebenso betheiligt wie Jobst Tetzel $=$ cromatfogel und Johann Feucht $=$ wiederumb, der als Schreiber diente. Gabriel Tetzel $=$ liligenstengel zog schon 1440 nach dem gelobten Lande: sehr luäufig wird er freilich erst seit 1474 in den gedruckten Berichten erwähnt. Ebenso tritt Wilhelın Derrer $=$ freimut häufiger erst seit 1471 hervor; aber er war doch auch schon 1458 consul junior. (Vgl. Würfel Nürnb. Stadt Gesch. I. 381.) Niklaus Gross $=$ grünling wird hauptsächlich in dem Prozesse ron Niklans Muffel $=$ golias im J. 1469 erwähnt. Losunger war er 1476-91; er wurde es an Stelle Anton Tuchers = schauloch, der dieses Aint 1461 erhielt (D. Stchron. X. 265), nachdem er schon 1459 neben Ludwig Pfinzing = gesell im Rathe sich als gegner Albrecht Achills gezeigt hatte. (D. Stchron. X. 243.) Uebrigens war Niklaus Gross schon 1450 neben Niklaus Muffel und Hans Müllner = tunckel in dem Streite Albrecht Achills mit Nürnberg thätig. (D. Stchron. II. 396. 397.) Seit 1462 war Rathsmitglied Peter Harsdürffer $=$ Rulandt, der in den nächsten Jahren auch als jüngerer Bürgermeister erscheint. Aelter war Meister. Heinrich Imhoff = maulwerff, der 1439 als Freischöffe erwähnt wird und 1449 gestorben zu sein scheint. Hans Merklein = cleeblatt ist nur als Kanzleischreiber erst 1491 nachweisbar, während Johann Muffel $=$ stentz und doctor Rott $=$ linckrutte schon in dem Muffelschen Prozess 1469 erwähnt werden. Von Jörg Spalter $=$ spelten vermag ich nichts Näheres anzugeben, doch gehörte er einer Nürnberger Familie an. Jörg Spengler $=$ jaspis ist jedenfalls der spätere Rathsschreiber, der schon beim burgundischen Kriege $1474 \mathrm{zum}$ „A usgeber" vorgeschlagen war. Ueber seine frühere Thätigkeit weiss ich nichts anzugeben. Nicht eigentlich zu den Nürnbergern gehört Ulrich Arzt = finckenau, da er 1446 von Nürnberg, wohin sein Vater ausgewandert war, wieder nach Augsburg gezogen ist. In seine Sachen verwickelt ist auch der Augsburger Bürger Gossenbrot $=$ geissbart neben dem Nürnberger Anton Baumgartner $=$ spinnenwebe. Ebenso ist hierbei

Tod angemerkt. A. a. O. II. 346. Ob es aber der hier gemeinte sei, bleibt zweifelhaft. 
nur mittelbar zu erwähnen Schüttensam = wurm; es ist fraglich, nb der jüngere Hans Schuttensam gemeint ist, der 1464 den Nürnbergern einen Feindsbrief zugesendet hat. Einige Personen, wie Hans Waltstromer $=$ kachel, Franz Waltstromer $=$ kechelein, Daniel Ulmer $=$ darnvol, kommen in dem Prozesse Peters von Argon vor. -

Ueber die „böcklergesellschaft in Beyren" $=$ pockstirn vernag ich keine weitere Auskunft zu geben, als dass sie 1467 aufgelöst werden sollte, aller Wahrscheinlichkeit nach also schon 1461/62 bestand. 1) Jedenfalls gehört der Name Hans Gewolff = geltnutz hierher, da ein Gewolf als Hauptmann des Böcklerbundes erwähnt wird. Die ,gesellschaft sant Jorgen schilt" $=$ hasel wird in dem Kampfe zwischen Albrecht Achill und Ludwig von Bayern suwobl im J. 1460 (Hasselholdt I. 155) wie auch 1461 (Ib. I. 175) erwähnt; die ,ritterschaft der neuen vereinung in Francken" $=$ vorhen wird gleichbedeutend sein mit der 1461 mehrfach erwähnten Ritterschaft der grossen oder kleinen Vereinigung in Franken.

Dass viele Reichsstädte (Augsburg $=$ troschel, Dinkelsbühl $=$ hering, Frankfurt $=$ krebs, Esslingen $=$ henn, Gingen $=$ ziella, Metz $=$ kartenspil, Nördlingen $=$ widhopf, Nürnberg = sampnung, Regensburg $==$ bücking, Rothenburg $=$ geyr, Rottweil $=$ pin, Strassburg = schnepf, Schaffhausen $=$ obermann von bertzen, Schweinfurt $=$ stul, Donauwörth $=$ all, Ulm $=$ colmeis, Windshein $=$ sittich, Weissenburg $=$ storch) genannt werden, würde an sich ohne Beweiskraft sein. Freilich sind grade 1461/62 sehr viele Städtetage, auf denen die genannten Städte vertreten waren, abgegehalten worden. Wichtiger schon ist es, dass etlich stett an Augspurg hangende $=$ swan und etlich stett an Ulm hangende $=$ zeislein und etlich stett an Augspurg und Ulm hangende $=$ lerch noch besonders erwähnt werden. Denn am 6. Februar 1462 wurden auf dem Tage zu Olm die Städte Augshurg, Dinkelsbühl, Nördlingen, Rothenburg, Schweinfurt, Donauwörth (allerdings auch noch dus hier nicht genannte Hall) dem Markgrafen von Brandenburg zugetheilt, während Ulm und die übrigen Städte dem Grafen Olrich von Württemberg überwiesen wurden. Aber auch andere reichstett $=$ küng von kleo wurden vom Kaiser dringend zur Hilfsleistung aufgefordert; so die reinisch stett $=$ rapp, auch die reichstett im

1) Hasselholdt-St. S. 19 versprach im 2. Bde. seines Werkes über Herzog Albrecht IV. daríber zu handeln, ist aber durch den Tod an der Vollendung seines Buches verhindert worden. - Vgl. auch Schmeller-Fr. I. 204. 
Elsass = kïng von hertzen, selbst die Schweizer = stockfisch blieben mit dieser Zunuthung nicht verschont (vgl. Hasselh. I. 228); deshalb wird Schaffbausen, die stat, die ein bruck über Rein hat = obermann von hertzen, werden die stett Freyburg im Uechtland, Bern, Soliter, Lucern $=$ pinstock angeführt sein. Dazu kommt, dass manche sonst selten erwähnte Städte gerade in dieser Zeit und in diesem Verzeichniss auftreten. So Lauingen (Laugingen) $=$ hohenstat, das in Dezember 1461 den Wittelsbachischen Fürsten, bez. ihren Räthen als Sammelpunkt diente und 1462 in Februar neben Heidenheim $=$ wasservalle rom Markgrafen $\Delta$ lbrecht Achilles angegriffen wurde; Kornneuburg ${ }^{1}$ ) = kornacker, das neben neuenstatt in Oesterreich $=$ gewitz sowohl 1461 wie 1462 erwähnt wird. Nicht lange vorher war der Kaiser in Gretz = gregler, dem lande auf der Steyrmark = bald, dem Lande ob der Enz $=$ au, in Linz $=$ Weisshofen gewesen; in Wien = rotttroschel wurde er 1462 belagert gehalten. Brichsenstadt (Pryssenstadt) $=$ Ofenhausen wurde 1461 vom Bischof von Würzburg belagert und 1462 erobert. Burghansen = sara und Lardshut $=$ - flaschland (Kloster zu Reun $=$ stiglitz ist jedenfalls Rain, wo Herzog Ludwig im Dezember 1461 weilte, Hasselholdt II. 578) waren die Residenzen Herzog Ludwigs; dagegen Feuchtwangen 2) $=$ abbtei, Schwabach ${ }^{3}$ ) $=$ stor, Kadolzburg ${ }^{4}$ ) $=$ Baryss, Neustadt a. d. Aisch 5 ) $=$ nachtrab, Kitzingen $\left.{ }^{6}\right)=$ unru Aufenhaltsorte Markgraf Albrechts in jener Zeit. Unter den Städten, die 1461 Herzog Ludwig dem Markgrafen abgewinnt, befinden sich die Stadt Bernheim 7 ) $=$ stockeul, Roth $=$ Reinpoldt, unter den Schlössern Hoheneck $=$ niderberg, ausser der schon oben erwähnten Neustadt.

Es bliebe nun noch zu erweisen, dass auch die sonstigen Ausdrücke und Redensarten auf die Verhältnisse der Jahre 1461/62 passen. Auf kriegerische Verhältnisse beziehen sich z. B. die Worte:

1) 1461 im Oktober sollte ein gittlicher Tag daselbt abgehalten werden. Hasselholdt I. 179.

2) Dort sammelte sich 1461 das Heer Mlbrecht Achilles. Hasselh. I. 147.

s) In Schwabach hielt sich Albrecht Achill z. B. 4 Wochen lang im Sept. 1461 auf. Hasselhol t I. 147.

4) Bei Kadolzb rg sammelte sich im Okt. 1461 das Heer der Bischöfe von Würzburg und Ba r berg. Hasselholdt J. 193.

5) 1461 von Her , $g$ Ludwig eingenommen.

9) Dort stand Albrecht Achill mit seinen Truppen im Okt. 1461. Hasselboldt I. 193.

7) Vgl. Höfler Ludwig v. Eyb S. 127 Anm. 24. 
so lang diese krieg vorhanden sind = rechgarn; alle, disen kriegsleufften verdacht weren $=$ amerallen; antreffent hilff orler kriegsleufft itzund vor augen $=$ jeger und viele andere. Auf die eigenthümlich schwierige Stellurg Nürnbergs innitten der Parteien deuten die Ausdrücke: swerlich allenthalben verläumet = rotterstorch; wo wege mochten funden werden, das willfarung geschebe und durch die vinger gesehen $=$ rottbegsteltz; wo es nit anders wolt sein. ee wollten sie den rat zusampt herzog Iudwigen zu veind haben = rotaglester; zu sorgen, dass des rats armleut, auch der kaufman beschedigt werden möcht, und manche andere hin. Dass der fischgall am kaiserlichen hof $=$ rumpf erwähnt wird, erklärt sich daraus, dass gegen die (sämmtlich in diesem Schlüssel erwähnten) Städte Augsburg, Nürnberg, Ulm, Weissenburg, Windsheim und Dinkelsbühl am 11. November 1461 (Hasselholdt I. 221) wegen Nichtbefolgung der kaiserlichen Befehle (sie waren vorher auf das höchst gepetten und ermant [ $=$ den himel zeigen] worden) auf Anrufen des kaiserlichen Kammer-Prokurator-Fiskals in die Pönen verfallen erklärt wurden, mit welchen das kaiserliche Ausschreiben (keiserlich gebot und verbotbrief $=$ greifen) die Ungehorsamen bedroht hatte.

Diese Beziehungen liessen sich noch viel weiter verfolgen; aber ich fürchte, es möchte allzu ermüdend werden, alle Einzelnheiten anzuführen. Nur die eine Bemerkung sei noch gestattet, dass manche Wendungen, wie lantfride $=$ gemein, reformarcion $=$ luchs auf allgemeinere, das Reich damals bewegende, Fragen sich beziehen, während andere wieder, wie z. B. statsteuer $=$ raub, neu spital $=$ gerstenacker sich nur auf besondere Nürnbergische Angelegenheiten deuten lassen. Daraus möchte zu folgern sein, dass dieser Schlüssel nicht blos für einen speciellen Zweck, sondern für längere Zeit gebraucht worden ist. Und das würde auf die Frage nach dem terminus ad quem führen.

Der Anhaltspunkte zur Beantwortung dieser Frage sind nur wenige. Der Grundstock des Schlüssels muss wohl vor den J. 1464 rorhanden gewesen sein, da sonst schwerlich Herzog Friedrich von Sachsen ausgestrichen und an seiner Statt Herzog Ernst eingetragen worden, da sonst nicht von der zweiten Hand erst Albrecht IV. von München, Graf Ott von Hennenberg, Kurfürst Philipp von der Pfalz, Herzog Albrecht von Sachsen hinzugefügt worden wären. Ueber welchen Zeitraum sich nun aber die Nachträge der zweiten Hand erstreckt haben, ist schwer zu sagen. Manches deutet darauf, 
dass der Schlüssel noch in der Zeit des Muffelschen Prozesses benützt worden sei; es ist doch zum mindesten auffällig, dass Dr. Seiffrid = boech, dessen Gutachten in Sachen Conrad Goldasts und Hans Müllers dabei erwähnt wird, nachträglich ebenso wie Jacob Roelinger, der erst seit 1468 in den Rathslisten geführt wird hinzugeschrieben worden ist. Auch der Ausdruck secklein $=$ mistlein ist auffällig, da Muffel des seckleins halb ein Geständniss ablegte. $J_{\mathfrak{a}}$, die Erwähnung des Nürnbergischen Feldhauptmanns im burgundischen Kriege Lutz Schott $=$ Hector würde auf einen noch späteren Gebrauch schliessen lassen, obwohl allerdings Lutz Schott auch 1462 wegen der Vertheidigung des Schlosses Weinsberg hätte erwähnt sein können (s. Stälin a. a. 0.); doch damit würde ein gefährliches und weites Gebiet der Vermuthung betreten, von dem ich mich lieber fernhalten will.

Den sichersten Beweis für die Zeit der Anwendung des Schlüssels zu erbringen, bin ich leider ausser Stande. Auf eine diesbezügliche Anfrage bein k. Kreisarchive zu Nürnberg wurde mir der Bescheid. dass Relationen Nürnbergischer Gesandten aus jenen Jahren nicht vorhanden seien, und da gerade aus dem J. 1461 auch das Briefbuch fehlt (vgl. D. Stchron. X. 264 Anm. 1), so ist und bleibt das Material zur Beurtheilung der hierher gehörigen Fragen lückenhaft. Aber sollte nicht doch einmal ein glücklicher Zufall die doch schwerlich vernichteten geheimen Berichte ans Tageslicht fördern? Dass sie nicht registrirt worden sind; würde durchaus erklärlich sein. Vielleicht finden sie sich gelegentlich einmal bei anderen Archivalien, gerade so wie ja auch dieser Schlüssel in spätere Aktenstiicke hineingerathen ist. 